\title{
Experiments on Steady State Particle Control in Tore Supra and DIII-D*
}

P. K. Mioduszewski, J. T. Hogan, L. W. Owen, R. Maingi, D. K. Lee, D. L. Hillis, C. C. Klepper, M. M. Menon, C. E. Thomas, T. Uckan, M. R. Wade Oak Ridge National Laboratory, Oak Ridge, TN 37830, USA

M. Chatelier, C. Grisolia, Ph. Ghendrih, A. Grosman, T. Hutter, T. Loarer, B. Pégourié CEA-Cadarache, F-13108 St. Paul lez Durance, France

\author{
M. A. Mahdavi, M. Schaffer \\ General Atomics, San Diego, CA, USA
}

\begin{abstract}
Particle control is playing an increasingly important role in tokamak plasma performance. The present paper discusses particle control of hydogen/deuterium by wall pumping on graphite or carbonized surfaces, as well as by external exhaust with pumped limiters and pumped divertors. Wall pumping is ultimately a transient effect and by itself not suitable for steady state particle exhaust. Therefore, external exhaust techniques with pumped divertors and limiters are being developed. How wall pumping phenomena interact and correlate with these inherently steady state, external exhaust techniques, is not well known to date. In the present paper, the processes involved in wall pumping and in external pumping are investigated in an attempt to evaluate the effect of external exhaust on wall pumping. Some of the key elements of this analysis are: (1) charge-exchange fluxes to the wall play a crucial role in the core-wall particle dynamics, (2) the recycling fluxes of thermal molecules have a high probability of ionization in the scrape-off layer, (3) thermal particles originating from the wall, which are ionized within the scrape-off layer, can be directly exhausted, thus providing a direct path between wall and exhaust which can be used to control the wall inventory. This way, the wall can be kept in a continuous pumping state in the sense that it continuously absorbs energetic particles and releases thermal molecules which are then removed by the external exhaust mechanism. While most of the ingredients of this analysis have been observed individually before, the present evaluation is an attempt to correlate effects of wall recycling and external exhaust.
\end{abstract}

* Research sponsored in part by the U. S. Department of Energy (USDOE) under the contract DE-AC05-84OR21400 with Martin Marietta Energy Systems, Inc., and under the International Collaboration agreement between the USDOE and the Association Euratom-CEA 


\section{Introduction}

Particle control is playing an increasingly important role in optimizing tokamak plasma performance. In the present paper, the discussion of particle control is focused on the control of the working gas, i. e. of hydrogen and/or deuterium. While there are many ways of particle control through control of the sources such as gas puffing, neutral beam fueling, or pellet fueling, particle balance also requires the provision of the corresponding particle sinks. In tokamaks with graphite walls or carbonized surfaces, this has been accomplished by conditioning the walls so as to provide net absorption of the incident particle flux for some period of time. Due to finite wall capacities, however, this technique is limited to transient pumping and pulsed operation. Therefore, methods are being explored that are suited for stationary pumping such as pumped limiters or pumped divertors. While some initial pumping experiments have been performed in the past on short-pulse experiments with pumped divertors or pumped limiters $[1,2,3,4]$, the pulse duration of these experiments was still short enough so that external particle sources could be balanced with wall pumping and the external particle control was mostly a demonstration of the principle rather than a necessity for controlling the density.

The future direction towards long-pulse or steady state experiments requires the use of pumped diverors or pumped limiters and a systematic understanding of their effect on plasma particle control and wall recycling. In this paper, we will present results on wall pumping provided by graphite or carbonized surfaces, as well as on external particle exhaust with pump limiters and pumped divertors. The eventual goal of this research is to develop techniques consisting of a combination of wall recycling and external purnping for steady state particle control in devices such as Tore Supra [5] or the planned Tokamak Physics Experiment (TPX) [6]. The present paper will, in particular, stress the role of charge-exchange fluxes on the core-wall-exhaust particle dynamics. 


\section{Wall pumping}

The term "wall pumping" is commonly used for the spontaneously occurring net particle removal by the wall during standard plasma operation, as well as for the induced pump-out effect that occurs when the plasma is suddenly moved to a part of the wall that is able to provide net particle absorption, usually the inboard wall. Recycling phenomena on graphite and carbonized surfaces seem to be similar [7] and in the following, we will not distinguish between the two different forms of carbon surfaces. Spontaneous wall pumping seems to be a prerequisite for good plasma performance in almost all tokamaks, by providing low neutral edge density. It is usually achieved by keeping the hydrogen inventory in the graphite wall low, either by operating at elevated wall temperatures $\left(>300^{\circ} \mathrm{C}\right)$, or by conditioning the wall with helium glow dicharges which removes the accumulated hydrogen between discharges. Under these conditions, the wall can usually absorb particle fluxes of the order of tens of Torr-L/s for several seconds, which suffices to balance the neutral beam fueling, or some external gas puffing.

The details of the effects of wall pumping or external particle exhaust on plasma performance are not fully understood. The induced wall pumping, caused by moving the plasma to the inboard wall, has been studied in detail for JET [8,9] and TEXTOR $[10,11]$, with emphasis on the possible mechanisms involved in the wall retention. In the present paper, the discussion will be focused on the inferred core-wall particle dynamics and processes in the SOL, deduced from the plasma profile evolution. No attempt will be made to explain the wall response under the variety of occurring circumstances with a comprehensive recycling model.

\subsection{Wall pumping experiment in Tore Supra}

\section{Experimental conditions}

In order to study the mechanisms involved in the core-wall particle dynamics, a wall pumping series in Tore Supra was analyzed. These experiments were described in more detail 
earlier [12,13]. After thorough wall conditioning by 60 hours of helium glow, a series of discharges was carried out with a common format. The experiment was conducted under the following conditions: deuterium was the working gas, the plasma current was $1.2 \mathrm{MA}$, the current flat-top was $8 \mathrm{~s}$ (starting at $\mathrm{t}=2 \mathrm{~s}$ ), and the toroidal field was $3.8 \mathrm{~T}$. The inboard wall consisted of graphite tiles. The entire wall was covered with a carbonization layer and was kept at a temperature of $180-200^{\circ} \mathrm{C}$. The discharges were initiated on the non-activated Outer Pump Limiter (OPL). After a pre-fill and plasma breakdown, gas puffing, lasting 3-4 seconds with flow rates between 3.75 Torr-L/s and 15 Torr-L/s ( 1 Torr-L $=3.2 \times 10^{19}$ molecules), was applied to achieve the desired plasmas. Approximately 2-3 seconds after the termination of the gas puff, the plasma column was shifted to the inner wall, and a rapid density decay after the plasma shift was observed.

\section{Density response}

This behaviour is shown in fig.1, which depicts the plasma density evolution of the first and last five discharges of the sequence of a total of 18 discharges. There appeared to be a shortterm and a long-term effect. In the short term, viz. during a single discharge, the wall pumping was strong at the time of the plasma shift, but saturated within less than one second. Then, between the discharges, the pumping surfaces must have become sufficiently depleted to pump again during the subsequent discharge. This indicated that for times in the order of the duration of the discharge (10s), a fraction of the pumped particles was retained, while during times between discharges (typically 900s), a considerable fraction was released, providing wall pumping again for the subsequent discharge.

The long-term effect of the observed wall pumping became obvious over the whole discharge sequence. As the density traces in fig. 1 indicate, the pump-out of the last five discharges in the series showed a diminished initial pumping rate and a change in the saturation behaviour. An estimate of the initial pumping rate was given by the initial decay rate $\left(\tau_{0}\right.$ $=\mathrm{N} /(\mathrm{dN} / \mathrm{dt}))$ of the plasma density as it was first moved to the inner wall. This decay rate varied 
from $0.5 \mathrm{~s}$ at the beginning of the sequence, to $2.5 \mathrm{~s}$ at the end. Thus, the qualitative trend was that the pumping rate decreased as a function of shot number. A quantitative estimate of the initial wall pumping rate yielded $\sim 1 \times 10^{21}$ particles/s and a total removal of $5 \times 10^{20}$ particles for the first discharge. Estimating a wetted area of $\sim 6 \mathrm{~m}^{2}$ of the total inner wall surface area of $10 \mathrm{~m}^{2}$, this corresponded to $\sim 8 \times 10^{19}$ particles $/ \mathrm{m}^{2}$, or approximately 10 equivalent monolayers.

While fig. 1 shows the global density response, given by the changes of the volumeaverage density, more details were revealed by examination of the electron density profile, obtained with Thomson scattering. The electron density profile of shot 5068 as a function of time is shown in fig. 2. This profile shows, in particular, that the edge density decreased as the wall pumping began. This edge density reduction appeared to be an important ingredient to the overall wall pumping mechanism, because it led to an increase of the neutral penetration depth, which changed intensity and energy spectrum of the charge-exchange particle flux. This is the basis of the model presented here.

\subsection{Model and data analysis}

The core-wall particle exchange model presented here, is based on the idea that chargeexchange reactions provide an important mechanism for wall pumping. Charge-exchange reactions have been identified to play an important role in impurity generation [14] as well as in wall recycling. In earlier studies, it was inferred that a mechanism existed by which up to $10 \%$ of the core efflux could be retained by strong inner wall pumping [15]. The flux of energetic charge exchange particles (e.g. with $\mathrm{E}>1 \mathrm{keV}$ ) was deposited deeply in an unsaturated region so that the flux to the surface could be retained in the wall for long times (tens of seconds), rather than recycling within a time scale of a plasma particle confinement time.

Plasma profiles and calculation of charge-exchange fluxes

To estimate the behavior of the charge exchange particle deposition in the a-C:H carboniza- 
tion layer [16] (covering the graphite tiles) as a function of time for each of the discharges in the sequence, detailed profile information was needed. This was necessary because the charge exchange efflux depended on the parameter $n_{i} n_{0}\langle\sigma v\rangle_{c x}$ as a function of $p, t$ (minor radius and time), with $n_{i}$ the ion density, $n_{0}$ the neutral density, and $\langle\sigma v\rangle_{c x}$ the rate coefficient for charge exchange reactions. The measured Thomson scattering profiles provided $n_{e}(\rho, t)$ and $T_{e}(\rho, t)$ and it was assumed that $T_{i}(\rho, t)=0.75 T_{e}(\rho, t)$ for the series of ohmic heating $(O H)$ cases presented here. This was found to be a reasonable assumption in an earlier analysis of a comparable sequence of $\mathrm{OH}$ discharges with a full radial transport code [15].

Given the profiles of $n_{e}(\rho, t), T_{e}(\rho, t)$ and $T_{i}(\rho, t)$, then the neutral particle distribution in the plasma had to be found. The SPUDNUT 1-D neutral transport code [17] was used for this. Because the core plasma was the region of interest, rather than the SOL or a pumping region, the use of a slab model rather than a 3-D code, such as DEGAS, was justified. The SPUDNUT code had to be modified in several respects in order to perform the calculation. The surface model had been changed to model the $\mathrm{D}->\mathrm{C}$ deposition, and the Tore Supra Thomson scattering profiles were spline-fit for use in the code.

\section{Surface reflection and deposition}

Since Tore Supra's major plasma-facing material is an a-C:H layer, rather than stainless steel, the surface data for carbon were included in the SPUDNUT model. Reflection data from the Eckstein database [18], with modifications at low energy derived from analysis of fractal surfaces by Ruzic [19] were used for this analysis. The wall deposition model in SPUDNUT uses a modification of the parametrization based on calculations by the Marlowe code [20] for stainless steel. This produces a fit to the depth distribution function which has been checked for the case of D->C by application of the TRIM-SP code [21] for several cases from this sequence. 
Measured charge-exchange fluxes during the wall pumping sequence

The measured charge exchange fluxes for shot 5068 showed the characteristics of charge exchange pumping directly. Figure $3 \mathrm{a}$ depicts the measured time dependence of the charge exchange flux for energies above $1 \mathrm{keV}$ as the plasma was moved to the inner wall and enhanced wall pumping began. Figure $3 \mathrm{~b}$ shows the energy spectrum before and after the plasma shift, with a strong increase in the energetic component $(\mathrm{E}>1 \mathrm{keV})$ of the charge exchange flux at the start of inner wall pumping. This increase had a Maxwellian character as a function of energy, indicating that it arose from the deeper penetration of neutrals to plasma zones of higher ion temperatures. In addition to the working gas deuterium, the figure also shows the charge exchange spectrum of hydrogen which originated from the residual wall inventory.

The corresponding behavior of the charge exchange spectrum, calculated with the SPUDNUT model using the measured $\mathrm{n}_{e}$ and $\mathrm{T}_{\mathrm{e}}$ profiles, is shown in fig. 4 . The figure shows the calculated charge exchange efflux for $\mathrm{E}>1 \mathrm{keV}$ as a function of time, indicating the change after the plasma shift. Figure $4 \mathrm{~b}$ depicts the calculated change of the charge exchange spectrum to higher energies: shown are the spectra for $t=4.3 \mathrm{~s}$ (before the plasma was moved to the inner wall) and at $t=6.5 \mathrm{~s}$ (after the plasma was moved to the inner wall). The trends and the magnitudes of the calculated changes were in good agreement with the experimental values, and this permitted further analysis of the corresponding processes which contribute to this agreement: the neutral penetration into the core and the deposition of energetic cx neutral efflux in the wall. The increase in the penetration length of neutrals into the core, due to the edge density decrease, resulted in a change of the neutral density profile as a function of radius. The evolution of the (normalized) neutral density profile in the core, calculated with the present model, is shown in fig. 5. The strong reduction in density allowed the penetration of neutrals to regions with $\mathrm{T}_{\mathfrak{i}}>1 \mathrm{keV}$, and the generation of an increased flux of higher energy particles to the wall as demonstrated in fig. 4. 
Calculated wall deposition of charge exchange flux during the sequence

In the present analysis, no attempt was made to apply a detailed wall recycling model. Instead, the plasma profile evolution was used as the basis for the analysis. Wall deposition profiles, derived from particle fluxes, compatible with the plasma profile evolution, were calculated with SPUDNUT and TRIM before and after the plasma shift. The result is shown in fig. 6 , indicating clearly a shallow and a deep component of the particle deposition. Figure 6a shows the total deposition profile, over the interval $0<\mathrm{d}<250 \AA$ in the a-C:H layer, and fig. $6 \mathrm{~b}$ restricts the range to a depth characteristic of high energy charge exchange deposition $20 \AA<d<250 \AA$. (Note that the scale of fig. $6 \mathrm{~b}$ is reduced by a factor 10 compared with Fig. 6a.) Assuming an approximate penetration depth of $0.6 \AA / \mathrm{eV}$ (for monoenergetic particles), which is consistent with the MoellerScherzer deposition model $[22,23]$, one finds that the bulk of the charge exchange flux was, as expected, due to the low energy Franck-Condon efflux and was deposited in a shallow layer at a depth around $10 \AA$. . However, a significant fraction (>10\%) was deposited at distances $>100 \AA$ in the a-C:H layer.

If this trend were to persist throughout the shot sequence, then the long term retention of the charge exchange flux would imply a recycling coefficient of $R \sim 0.9$ for extended pulse lengths. However, during the course of the sequence, the deep deposition profile was found to be strongly reduced. This was thought to be due to the decrease of the predominant deposition in the shallow layer which increased the recycling, raising the plasma density in the edge region and increasing the core plasma opacity to neutral penetration. Thus, wall-evolved neutrals no longer had access to the more energetic ion distribution. This trend was seen during the entire shot sequence. Figure 7 shows the corresponding electron density profile evolution for the last discharge of the series, shot 5085. It is obvious that the decrease in edge electron density at the time of the shift to the inner wall was significantly less than for the corresponding case of the first shot shown in fig. 2 . 
Scenario for induced wall pumping

The overall scenario for charge exchange pumping is the following: (1) Initially, the inner wall retains a fraction of the incident plasma and recycling Franck-Condon flux in a shallow layer $(<20 \AA)$, which decreases the plasma edge density. (2) As a consequence, the recycling neutrals, which consist mostly of Franck-Condons and a small fraction of directly reflected neutrals, can penetrate deeper into the plasma edge. (3) Due to the deeper penetration of neutrals, the spectrum of charge exchange particles is shifted to higher energies and the energetic flux $(\mathrm{E}>1 \mathrm{keV})$ increases. (4) At higher energies, the charge exchange particles are retained in deeper, unsaturated wall surface layers. (5) As the shallow deposition layer saturates due to high primary ion and Franck-Condon fluxes, recycling increases and causes a rise in the plasma edge density. (7) Due to the edge density rise, the depth of neutral penetration is decreased, and the maximum value of $T_{i}$ which is reached by edge neutrals also decreases, and thus the range of deposition in the wall also becomes shallower, eventually terminating the charge exchange pumping process.

One can speculate that continuous external particle exhaust would keep the plasma edge density continuously low, thus increasing the energetic charge exchange flux and extending the wall pumping capacity by about an order of magnitude by filling the deeper layer. To investigate how external pumping affects the core-wall particle dynamics, particle exhaust experiments with external pumping are discussed next.

\section{Particle exhaust with external pumping}

Particle control experiments with external exhaust have been performed in a number of devices with pumped limiters $[2,3,4,24]$ and pumped divertors $[1,25,26,27,28]$. Pumped divertor experiments on JET [29] are planned to begin in the second half of 1994 . In contrast to the transient wall pumping effects, the long-pulse external exhaust experiments have shown that the density could be controlled under stationary conditions. In past experiments on smaller tokamaks with stainless steel walls, it was observed that approximate particle balance could be 
obtained by consideration of external fueling and particle exhaust. Exhaust experiments on present, graphite-dominated machines, however, indicate that fueling as well as exhaust can be totally dominated by the wall providing a particle source or sink respectively. In the following, we will describe some particle exhaust experiments with pump limiters in Tore Supra and pumped divertor experiments in DIII-D. While pump limiters are presently not the preferred option for power and particle handling in future machines, they provide a method for external particle exhaust similar to that of divertors, permitting studies of the interaction between external exhaust and corewall particle dynamics

\subsection{Exhaust experiments in Tore Supra with moderately conditioned walls}

Tore Supra was designed with the objective to develop steady state plasma operation [30]. The particle handling system consists of the standard gas puffing system, a centrifuge pellet injector which is capable of injecting up to 100 pellets [31], a system of six bottom and one outboard pump limiter (OPL) $[32,33]$, and an ergodic divertor with particle exhaust capabilities $[34,35]$.

\section{Experimental conditions}

Since the wall particle inventory in graphite machines usually dominates the plasma inventory by more than an order of magnitude, the wall conditioning state is an important parameter in exhaust experiments. The following experiments were performed after an over-night helium glow conditioning session. Helium glow wall conditioning between shots, as performed in some other devices, is presently not being performed in Tore Supra; it has not been effective, most likely because of the permanent toroidal field resulting from the superconducting magnetic coils. The present experiment consisted of a series of ohmic discharges with 1.5 MA plasma current, $4 \mathrm{~T}$ toroidal field, deuterium prefill and gas puff, and a wall temperature of $\sim 150^{\circ} \mathrm{C}$ [36]. In addition to the standard core diagnostics, the OPL diagnostics included an infrared camera to determine the 
heat deposition profile, Langmuir probes in the throat for electron density and temperature as well as decay length measurements, and pressure gauges in the pumping plenum.

\section{Density control}

The effect of external pumping depends to a large extent on the conditioning of the walls. The results of external exhaust with previously conditioned walls in Tore Supra is demonstrated in fig. 8, showing time traces of discharges 10449 with pump limiter "off" and 10450 with pump limiter "on". The gas puff traces for these two discharges are shown in fig. 8a, the volumeaverage densities in fig. 8b, and the pump limiter pressures in fig. 8c. Discharge 10499 was the tenth discharge of the day and the wall conditioning effect was no longer strong but, nevertheless, clearly effective. The pre-puff for these discharges was 10 Torr-L. Gas puffing was applied between $t=0$ and $t=5 \mathrm{~s}$, resulting in a total amount of 82 Torr- $L$ of gas. Since the resulting plasma inventory was only 15 Torr-L, the global wall condition must have been in a pumping state. Nevertheless, when the gas was turned off at $t=5 \mathrm{~s}$, the density decay was relatively slow: $\tau_{\mathrm{p}}{ }^{*}$ at $t=5 s$ was $20 \mathrm{~s}\left(\tau_{\mathrm{p}}{ }^{*}=\tau_{\mathrm{p}} /(1-\mathrm{R})\right.$, with $\tau_{\mathrm{p}}$ the particle confinement time and $\mathrm{R}$ the recycling coefficient). In comparison, the pre-puff for the pumped discharge 10450 was identical and the gas puff was programmed to achieve the same line-average density $\left(7 \times 10^{19} \mathrm{~m}^{-2}\right)$ as the nonpumped discharge. (Since the volume-average density is shown here, profile effects made the densities slightly differ from each other.) After the end of the gas puff, the density of the pumped discharge decayed rapidly with $\tau_{\mathrm{p}}{ }^{*}=2.5 \mathrm{~s}$, indicating the strong exhaust effect of the pump limiter.

While the gas required during the density build-up differed only slightly for the two discharges, the gas puff rate during the phase of constant density, viz.between $t=3 s$ and $t=5 s$, was significantly higher for the pumped discharge. The total gas required for discharge 10450 was 132 Torr-L, compared to 82 Torr-L for discharge 10449. Assuming similar wall recycling for two consecutive shots, the extra gas was balanced by the pump limiter exhaust. 
The pressure in the pump limiter plenum reached 10 mTorr for the non-pumping case (the baratron gauge was actually saturating at $10 \mathrm{mTorr}$ ), while in the pumped case, the pressure at the pump dropped to approximately $1 \mathrm{mTorr}$ at a measured pumping speed of $23,000 \mathrm{~L} / \mathrm{s}$. The pumping speed of the titanium pump was inferred from the pressure gradient, measured with two gauges in the plenum and the known conductance between them.

\section{Particle balance}

The particle balance for discharges 10449 and 10450 was evaluated by dividing the shot into three parts: (1) the build-up phase (0-3s), (2) the stationary phase (3-5s), and (3) the density decay phase (5-8s). In the non-pumped discharge (10449), the total number of particles was shared by the plasma and the wall. Most of the gas input of 82 Torr-L was absorbed by the wall. A possible maximum of $\sim 15$ Torr- $L$, which corresponded to the plasma content at the end of the shot, could have been pumped out after termination of the shot. The particle balance for the pumped discharge (10450) is shown in fig. 9, with flow rates in Torr-L/s on the left-hand ordinates and total particle numbers in Torr-L on the right-hand ordinates. Figure 9a shows the fueling rate and integrated gas input, $9 \mathrm{~b}$ the change in plasma content and the total plasma inventory, $9 \mathrm{c}$ the exhaust rate and the integrated exhaust, and $9 \mathrm{~d}$ the wall pumping rate and integrated wall inventory. The wall balance was obtained from the equation $\mathrm{dN}_{\text {wall }} / \mathrm{dt}=\Phi_{\text {gas }}$ $V d n_{e} / d t-p S$, i. e. by subtracting the plasma content and the external exhaust from the gas input.

During phase (1) the exhaust was minimal due the low pressure and most of the gas was absorbed by the wall, similar to the non-pumped discharge. In phase (2) the difference in the fueling rate between 10450 and 10449 was balanced by the external exhaust. During phase (3) the gas puff had been turned off and the exhaust rate greatly exceeded the decay rate of the plasma. The only plausible source for this extra gas, which was removed in addition to the gas from the density decay, was the wall inventory. It has been known that graphite walls can provide strong fueling to the plasma, in addition, the present result indicated that, combined with external exhaust 
techniques, the wall could also directly supply particles to the exhausted gas. From a different perspective, external particle exhaust not only controls the particle content of the plasma, but also controls the wall inventory. This effect indicates possibilities for continuous, simultaneous wall conditioning during a discharge with external exhaust.

It seems to be plausible that the effect of the pump limiter on the wall inventory would be even stronger with walls that were not pre-conditioned, but rather in a state of relatively high hydrogen loading. This was studied in some detail as well, but due to space limitations, only the result will be quoted here. In a 10-s discharge, the wall inventory of an unconditioned wall was strongly depleted by the pump limiter [24]. Integrated over the whole discharge, the total particle exhaust was 75 Torr-L/s, while the only external gas source was a pre-fill of 5 Torr-L, i. e. the net reduction of the wall inventory was $70 \mathrm{Torr}-\mathrm{L}$ in one discharge. A quantitative measure of the wall conditioning effect due to the particle exhaust was the reduction of $\tau_{\mathrm{p}} *(-\mathrm{N} /(\mathrm{dN} / \mathrm{dt})$ at $t=5 \mathrm{~s})$ from $48 \mathrm{~s}$ to $5 \mathrm{~s}$ after only three pumped discharges. To achieve densities (without pumping) comparable to the ones before the pumped discharges, the prefill had to be raised to 15 Torr- $\mathrm{L}$, and a gas puff of 4 Torr- $L / s$ had to be applied between during $t=0-5$ s.

\subsection{Exhaust experiments in DIII-D with conditioned walls}

While correlations between external exhaust and wall conditions were first studied on Tore Supra with a pump limiter, similar observations have since been made on the divertor tokamak DIII-D. The DIII-D tokamak was equipped with a baffle and a cryo-pump [37, 38, 39] to control the density of $\mathrm{H}$-mode discharges independently from the current and also to provide low-density target plasmas for current drive. First particle exhaust experiments were carried out to investigate density control [28] and to characterize low-density SOL and divertor plasmas achieved by divertor pumping [40]. While the speed of the cryo-pump could not be controlled, i. e. the pump was either "on" or "off", the resulting exhaust could still be controlled by variation of the distance between the divertor strike point and the pumped baffle. Excellent control of the density in 
ELMing H-modes with the pumped divertor has since been achieved [41]. With optimum pumping, the density could be decreased by a factor of two.

\section{Experimental conditions}

In DIII-D the walls are usually at room temperature during operation. Helium glow wall conditioning (HeGWC) is applied between discharges, which provides strong wall pumping and is a prerequisite for good H-mode performance [42]. These conditions provide an opportunity to study the particle balance with external exhaust and strong wall pumping. A series of pumping experiments was performed with the following conditions: ohmic target plasma during $t=0-1.5 \mathrm{~s}, 5$ MW neutral beam injection starting at $\mathrm{t}=1.5 \mathrm{~s}$, inducing the transition from L-mode to $\mathrm{H}$-mode, 1 MA plasma current, $2.1 \mathrm{~T}$ toroidal field, and $6 \times 10^{19} \mathrm{~m}^{-3}$ maximum line-average plasma density without pumping. After the density reached its maximum value of $6 \times 10^{19} \mathrm{~m}^{-3}$, the divertor strike point was moved at $t=2 \mathrm{~s}$ to the position of maximum pumping. At a pumping speed of approximately $30,000 \mathrm{~L} / \mathrm{s}$, the pressure in the baffle was $1 \mathrm{mTorr}$.

\section{Particle Balance}

The particle balance for a typical pumped divertor discharge (78012) is shown in fig. 10 . As in the particle balance figures above, the broken lines represent particle flow rates in Torr-L/s, indicated on the left-hand ordinate, while the solid lines represent integrated particle numbers in Torr- $\mathrm{L}$, indicated on the right-hand ordinate. The particle balance for this discharge had the following characteristics: during the plasma build-up between $t=0 \mathrm{~s}$ and $\mathrm{t}=1.5 \mathrm{~s}$, only $10 \%$ of the gas was consumed by the plasma, a negligibly small fraction was pumped, and almost $90 \%$ was deposited in the wall. After the H-mode was fully developed, at $t=2 \mathrm{~s}$, the outer divertor strike point was moved to the maximum pumping position and the particle exhaust increased to 30 Torr$\mathrm{L} / \mathrm{s}$, causing the plasma density to decrease. The observed exhaust rate (measured pressure $\mathrm{x}$ pumping speed) exceeded the sum of the external fueling plus the density decay rate, so that the extra exhaust had to be supplied by particles extracted from the wall, similar to the Tore Supra 
result discussed above. In addition, it showed that, with a sufficiently strong exhaust, the wall supplied particles to the pump even after prior wall conditioning and with extra particles provided by a continuous, external fueling source. From the behaviour of the wall inventory decrease, as shown in fig. 10 (d), it was not possible to conclude if a sufficiently long pulse would remove all the gas deposited during the plasma build-up phase, or if some gas would be retained in equilibrium.

The most dramatic example of wall depletion by external exhaust is shown in a second example which demonstrated that, even with external neutral beam fueling of $10 \mathrm{Torr}-\mathrm{L} / \mathrm{s}$, the divertor exhaust could diminish the wall inventory sufficiently to leave the wall more depleted after the discharge than it was at the start. In this case, the wall conditioning before the shot was performed with a mixture of helium and deuterium, so that the wall had a higher deuterium loading at the onset. This example is shown in fig. 11, in which the labels and parameters are the same as fig.10. In this case, all the gas absorbed by the wall during the build-up phase of the plasma was removed by the exhaust, and the wall loading actually crossed the zero line, indicating a higher degree of depletion at the end of the discharge than before the start.

\section{Analysis and modeling}

For the development of steady state particle control techniques, continuous external exhaust is a prerequisite. The present study investigates how external exhaust interacts with wall pumping and how a combination of these effects can lead to continuous particle control.

\subsection{Changes of $\tau_{p}{ }^{*}$ in the pump limiter sequence}

A simple measure for the comparison of the wall recycling state is a comparison of the $\tau_{\mathrm{p}}{ }^{*}$ values, evaluated at a given time, for the discharges in question. The effect of wall depletion by external exhaust was first assessed by analyzing the cumulative effect of six pump limiter discharges on the wall state. This analysis consisted of a comparison of the $\tau_{\mathrm{p}}{ }^{*}$-values after 
termination of the gas puff at $t=5 \mathrm{~s}$ in the sequence with moderately conditioned walls in Tore Supra. (In discharges with "moderately" conditioned walls, gas puffing is needed to build up the plasma.) Discharge 10449, which was not pumped, provided the reference value. It was succeeded by the six pumped discharges $10450-10455$, which served to condition the wall. This sequence of pumped discharges was then followed by shot 10456 which was not pumped. The non-pumped discharges 10449 and 10456 had similar densities, so that a direct measure of the difference in the wall recycling state was provided by a comparison of $\tau_{\mathrm{p}}$.

This series was also part of a density scan, so that a direct comparison of the $\tau_{\mathrm{p}}{ }^{*}$-values of all discharges required a normalization of the densities to their value at $t=5 \mathrm{~s}$. This is demonstrated in fig. 12 , which shows the decay rates, with $\tau_{\mathrm{p}}{ }^{*}=20$ s for the last shot before pumping, followed by $\tau_{\mathrm{p}}{ }^{*}=2.5 \mathrm{~s}$ for the first pumped discharge, and finally $\tau_{\mathrm{p}}{ }^{*}=3.5 \mathrm{~s}$ for the first non-pumped shot after the pumping sequence. After six pumped shots, with gas puffing between $t=0 \mathrm{~s}$ and $5 \mathrm{~s}$, the wall had been conditioned to a state where its pumping capacity was comparable to the external exhaust. Relating $\tau_{\mathrm{p}}$ * of the two non-pumped discharges 10449 and 10456 should be a reasonable comparison, because their densities were close to each other, $\left\langle\mathrm{n}_{\mathrm{e}}\right\rangle=4.4 \times 10^{19} \mathrm{~m}^{-3}$ and $5 \times 10^{19} \mathrm{~m}^{-3}$ respectively (the gas puff was programmed to produce identical line densities).

Since the densities of the whole series varied between 2.3 and $5 \times 10^{19} \mathrm{~m}^{-3}$, the $\tau_{\mathrm{p}} *$ values of the entire series did not directly reflect the wall state, because they contained $\tau_{p}$ which, presumably, depended on the density. A fair comparison of the $\tau_{\mathrm{p}}{ }^{*}$-values of the whole series would require a normalization with respect to $\tau_{\mathrm{p}}$. Since the particle confinement times of the concerned discharges were not known, the density dependence was inferred from modeling and previous experience. For edge-fueled, ohmic plasmas, a simple, analytic model by Engelhardt and Feneberg provides an inverse scaling of $\tau_{p}$ with density [43]. Experimentally, this inverse scaling has been found on several machines, as discussed e. g. in [44, 45, 46]. At fairly low densities, however, $\tau_{p}$ has been found on some devices to increase with density to a maximum and then, at 
higher densities, to decrease again inversely proportional with density [47]. Since the lowest $<n_{e}>$ in the present case was still $2.3 \times 10^{19} \mathrm{~m}^{-3}, \tau_{\mathrm{p}}{ }^{*}$ was normalized by assuming $\tau_{\mathrm{p}} \sim 1 / \mathrm{n}_{\mathrm{e}}$, and $\tau_{\mathrm{p}}{ }^{*} / \tau_{\mathrm{p}}$ proportional to $\tau_{\mathrm{p}}{ }^{*} n_{\mathrm{e}}$. Consequently, the values of $\tau_{\mathrm{p}}{ }^{*}<n_{\mathrm{e}}>$ are plotted for the whole series in fig. 13. This representation of the change of the wall state showed indeed a strong effect of the pump limiter exhaust during the pumped discharges and a slightly decreased effect after the pump was turned off.

\subsection{Detailed modeling of the pump limiter sequence}

To gain more insight in the observed phenomena, a series of pump limiter discharges without wall conditioning was modeled with two different approaches. In the first approach, the emphasis was on the core-wall particle dynamics. The model was constructed by using a combination of a 3-D Monte Carlo neutrals code, a 1-D radial plasma transport code, and a 1-D code describing the diffusion of hydrogen in the carbonized wall surface layer [15]. The second approach focused on the processes in the SOL and the pump limiter throat, with particular emphasis on ionization effects in the SOL. This model was based on a combination of the DEGAS neutrals code and Stangeby's analytic model for ionizing SOLs [48]. The major conclusions of the detailed modeling analysis were: (1) charge-exchange fluxes to the walls around the limiter played a crucial role in the core-wall particle dynamics due to the generation of thermal neutrals recycling from the wall, and (2) the ionization of the recycling thermal neutrals within the SOL led to direct exhaust of particles originating from the wall. The main ideas of the modeling analysis will be described next.

\section{Core-wall particle dynamics}

In the pump limiter discharges the limiter received a large fraction of the ion flux, and the refluxing neutrals created a neutral particle source in the core plasma near the limiter. Chargeexchange between neutrals in this localized cloud and the core plasma ions created a strong flux of charge exchange neutrals in the region around the limiter. This charge exchange flux was 
implanted in the a-C:H layer and then reemitted in an amount, and on a time scale, which was determined by the particle energy and the diffusion rate in the wall. To construct the model, quantitative estimates were needed of (1) the core efflux to the limiter, (2) the recycled flux of neutrals to the core, (3) the charge exchange flux of these neutrals incident on the wall near the limiter, (4) the recycled flux of hot and cold neutrals from the wall, and the flux extracted by the pump limiter. These fluxes were obtained by using measured plasma core, SOL, and exhaust data, and calculating the remaining parameters with a combination of a radial transport code, a 3-D Monte Carlo neutrals code for the core charge exchange efflux, a 1-D code describing the diffusion of hydrogen in the carbonized layer, and a 2-D version of the DEGAS neutrals code to determine details of SOL properties such as flux amplification factors [48]. A detailed description of this model and the involved particle fluxes is given in [15].

Under these conditions, a detailed balance of the particle fluxes was constructed for the non-pumped and the pumped discharges. As a typical example, the particle fluxes for a pumped Tore Supra discharge (shot 3127 , no prior wall conditioing) is shown as a function of time in fig. 14. Figure 14a depicts the ion fluxes from the plasma core, and fig. 14b the balance of the wall fluxes. (The "plate" fluxes refer to the fluxes to and from the neutralizer plate in the pump limiter throat.) Flux amplification factors of approximately two each were calculated with DEGAS in the SOL near the limiter and in the limiter throat, explaining that the limiter flux is approximately twice as high as the core efflux in fig. 14a. The result which was most significant for the present analysis was the large charge exchange particle flux, which was found to be comparable in magnitude to the core plasma efflux $(\sim 40$ Torr- $\mathrm{L} / \mathrm{s})$. While the charge exchange fluxes did not change the plasma particle balance, they generated recycling molecules which were predominantly thermal and were to a large extent ionized in the SOL. This ionization in the SOL was quantified in more detail in the second modeling approach. 
Ionizing scrape-off layer

For the second approach, the model was constructed by iterating Stangeby's formulation for ionizing SOLs with DEGAS calculations to obtain an approximately self-consistent description of the plasma/neutral transport within and near the outboard pump limiter $[48,49]$. The model was constrained by the following parameters: density and temperature values at the last closed flux surface from Thomson scattering; density, temperature and scale lengths in the pump limiter throat from Langmuir probes; and power flux scale lengths on the limiter face from infrared camera images. The modeling results reproduced all available experimental data for this discharge. The total recycling flux at the limiter neutralizer plate was that required to give the measured neutral plenum pressure. The converged values of the SOL decay lengths and the flux amplification factors produced detailed particle balance in the core, SOL and pump limiter. In this case, the result which was most significant for the present investigation of wall depletion, was the high ionization probability of recycling neutrals in the SOL. Stronger ionization in the SOL can be expected when the electron temperature exceeds $20 \mathrm{eV}$, because the direct ionization of molecules becomes more likely than Franck-Condon processes at higher temperatures [50]. The distribution of the ionization in the SOL is depicted in fig. 15 which shows the radial profile of the average $\mathrm{H}^{+}$ source rate resulting from 7.5 Torr- $\mathrm{L}$ thermal $\mathrm{H}_{2}$ originating at the vacuum vessel wall. The curve shows the first generation ions generated from the wall efflux and was the key result in understanding the effect of external pumping on the wall inventory. The maximum of the ionization curve was close to the pump limiter throat entrance, suggesting the possibility for the corresponding fraction of the ions, generated in the SOL, to flow directly into the throat.

\section{Discussion}

The analysis of the wall pumping sequence as well as the external exhaust experiments have pointed out the importance of charge exchange fluxes for the core-wall particle dynamics. How is external particle exhaust likely to interact with induced wall pumping in the present model? A combination of continuous external particle exhaust with induced wall pumping can possibly 
extend the deep-deposition pumping by keeping the edge density low and, hence, increasing the neutral penetration into the plasma core, leading to hardening of the charge exchange spectrum and to deep wall deposition. However, this can only last until the deep deposition is saturated and will not lead to steady state deep deposition pumping. The main feature of the deep deposition should be the long retention time which is assumed to be in the order of tens of seconds. If the deep deposition is maintained for this time by external exhaust, then the eventual saturation of deep deposition should be indicated by a change in the apparent wall response. The pulse lengths of the present experiments $(<10$ s) are not long enough to test this model, but planned experiments in Tore Supra should come close in the near future.

For the interaction of the external exhaust with spontaneous wall pumping, the following picture emerges: (1) Energetic charge exchange fluxes traverse the SOL and impinge on the walls with subsequent release of secondary particles, predominantly thermal molecules. (2) A large fraction of these recycling particles (65\% in the present case) becomes ionized within the SOL and typical fractions of $10-20 \%$ are removed by the pump limiter or divertor. This way, particles can follow a direct path from the wall to the pump limiter, decoupling the external exhaust to some degree from the plasma core. Since the experimental evidence indicates that the wall becomes depleted by this process, the recycling coefficient must be larger than unity under some conditions. This is conceiveably due to particle-induced desorption caused by energetic charge exchange particle fluxes. Due to the observed wall depletion, it is not obvious if the equilibrium state of the wall is a saturated or a depleted state. In either case, the wall will absorb fast charge exchange particles and release thermal molecules, in equilibrium with the wall temperature. Since a fraction of these particles is removed from the SOL, the apparent recycling at the last closed flux surface can be less than unity, even with the wall recycling being unity.

Since plasma, wall, and external exhaust form a dynamic system, the often quoted "wall equilibration time" is of less importance in a system with external exhaust. The wall equilibrium 
changes as a function of plasma parameters, especially charge exchange fluxes, and exhaust conditions. As shown above, during a long-pulse discharge, the wall inventory can increase or decrease. The relevant parameter is the system response time for density changes which is controlled by the global particle confinement time $\tau_{\mathrm{p}}{ }^{*}=\tau_{\mathrm{p}} /(1-\mathrm{R})$. For a given particle confinement time $\tau_{p}, \tau_{p}{ }^{*}$ is determined by the total recycling coefficient $R=\left(R^{\prime}-\varepsilon\right)$, where $R^{\prime}$ characterizes the wall and $\varepsilon$ the external exhaust. If $\left(1-\left(R^{\prime}-\varepsilon\right)\right)$ is sufficiently larger than $\left(1-R^{\prime}\right)$, then the external exhaust determines $\tau_{\mathrm{p}}$, and, hence, the density response time.

While the present analysis was focused on pump limiter discharges, the experimental evidence indicates that the same mechanism is effective in divertor discharges. In this case, the charge-exchange fluxes are likely to impinge on the wall areas around the divertor strike points. To investigate this, systematic experiments of wall saturation and subsequent depletion with external exhaust are planned for the near future in the DIII-D tokamak. Particle control issues on longer pulse lengths will be addressed in Tore Supra with experiments combining pump limiter exhaust and lower hybrid current drive, with expected pulse lengths of tens of seconds. This combination of experiments with strong exhaust, extended pulse-length, and multi-approach modeling is aimed at contributing to the data base for the development of steady state particle control scenarios for future fusion devices.

\section{Acknowledgements:}

The authors are indepted to the Tore Supra and DIII-D operation groups. Continuous encouragement and support for this collaborative effort from the U. S. Department of Energy, represented by E. Oktay, is gratefully acknowleged. 


\section{References:}

[1] F. Wagner and ASDEX-Team, Proc. of the IAEA Technical Committee Meeting on Divertors and Inpurity Control, Garching, 1989, p. 40

[2] P. K. Mioduszewski, et al., J. Nucl. Mater. 121 (1984) 285

[3] A. Grosman, and TFR Group, J. Nucl. Mater., 145-147 (1987) 819

[4] D. Goebel, R. W. Conn, W. J. Corbett, et al., J. Nucl. Mater. 162-164 (1989) 115

[5] M. Chatelier, in "Atomic and Plasma-Material Interaction Processes in Controlled Thermonuclear Fusion", R. K. Janev and H. W. Drawin (editors) 1993 Elsevier Science Publishers

[6] D. Hill, et al., these Proceedings

[7] E. Vietzke and V. Philipps, Nucl. Instr. and Meth. B23 (1987) 121

[8] J. Ehrenberg, J. Nucl. Mater. 162-164 (1989) 63

[9] J. Ehrenberg, P. J. Harbour, Nucl. Fusion, 31 (1991) 287

[10] J. Winter, J. Nucl. Mater. 145-147 (1987) 131

[11] J. Winter, J. Nucl. Mater. 161 (1989) 265

[12] C. Grisolia, P. Ghendrih, B. Pegourie, et al., J. Nucl. Mater. 196-198 (1992) 281

[13] C. Grisolia, T. Hutter, B. Pegourie, 18th EPS, Berlin 1991, Contr. Fusion Plasma Physics III-15C (1991) 57

[14] H. Verbeek and the ASDEX Team, J. Nucl. Mater. 145-147 (19877) 523

[15] J. T. Hogan, P. Mioduszewski, L. Owen, et al., J. Nucl. Mater. 196-198 (1992) 1089

[16] J. Winter, H. G. Esser, P. Wienhold, V. Phillips, et al., Nucl. Instr. Methods (1987) 538

[17] K. Audenaerde, G. Emmert and M. Gordinier, J. Comput. Phys. 34 (1980) 268

[18] W. Eckstein, Atomic and Plasma-Material Interaction Data for Fusion, Nucl. Fusion Supplement, LAEA, Vienna, 1991

[19] D. N. Rusic, H. K. Chiu, J. Nucl. Mater. 162-164 (1989) 904

[20] M. T. Robinson, I. M. Torrens, Phys. Rev. 39 (1974) 5008

[21] J. P. Biersack, W. Eckstein, Appl. Phys. 34 (1984) 73

[22] W. Moeller and B. M. U. Scherzer, J. Appl. Phys. 64 (10) 4860

[23] W. Moeller, J. Nucl. Mater. 162-164 (1989) 138

[24] C. C. Klepper et al., J. Nucl. Mater. 176-177 (1990) 708

[25] G. Haas, et al., J. Nucl. Mater. 121 (1984) 151

[26] M. Kaufmann and ASDEX-U Team, Proceedings of the 20th EPS Conference, Lisboa, Portugal, 1993

[27] R. Decoste, B. Terreauit, J. L. Lachambre, P. Couture, et al., in "Plasma Physics and Controlled Nuclear Fusion Research 1992, Vol.1, p. 383, IAEA, Vienna, 1993 [28] M. A. Mahdavi, et al., Proceedings of the 20th EPS Conference, Lisboa, Portugal, 1993 
[29] P.-H. Rebut and JET Team, Proc. IEEE 13th Symposium on Fusion Engineering, Knoxville, October 1998

[30] Equipe Tore Supra, in "Plasma Physics and Controlled Nuclear Fusion Research 1988, Vol.1. p. 9, IAEA, Vienna, 1989

[31] A. Geraud, C. Foster, et al., Proceedings of the 19th EPS Conference, Innsbruck, Austria, June 1992

[32] M. Chatelier, et al. in "Plasma Physics and Controlled Nuclear Fusion Research 1992, Vol.1, p. 341, LAEA, Vienna, 1993

[33] T. Loarer, T. Uckan, M. Chatelier, et al. J. Nucl. Mater. 196-198 (1992) 1078

[34] A. Grosman et al., these Proceedings

[35] T. Loarer et al., these Proceedings

[36] T. Loarer et al., Proceedings of the 20th EPS Conference, Lisboa, Portugal, 1993

[37] M. A. Mahdavi, M. J. Schaffer, P. Mioduszewski, et al., Report GA-A19547, Feb. 1989

[38] P. Mioduszewski, et al., J. Nucl Mater 176-17 (1990)

[39] M. M. Menon et al., Fusion Technol. 22 (1992) 356

[40] R. Maingi, P. K. Mioduszewski, M. A.Mahdavi, et al. this conference

[41] M. A. Mahdavi, et al., Proc. 15th IEEE/NPSS Symp. on Fusion Engineering, MA, 1993

[42] G. L. Jackson, T. S. Taylor, S. L. Allen, et al., J. Nucl. Mater. 162-164 (1989)489

[43] W. Engelhardt, W. Feneberg, J. Nucl. Mater. 76-77 (1978) 518

[44] R. Simonini, J. Spence and P. C. Stangeby, Plasma Physics and Controlled Fusion, 33 (1991) 653

[45] N. Ohyabu, N. H. Brooks, K. H. Burrell, J. C. DeBoo, S. Ejima, et al., J. Nucl. Mater. 121 (184) 157

[46] D. N. Ruzic, D. B. Heifetz and S. A. Cohen, J. Nucl. Mater. 145-147 (1987) 527

[47] A. J. Wootton, M. E. Austin, R. D. Bengsten, et al., Plasma Phys. Controll. Fusion 30 (1988) 1479

[48] L. W. Owen, M. Chatelier, J. T. Hogan, et al., J. Nucl. Mater. 196-198 (1992) 1125

[49] P. C. Stangeby, J. Nucl. Mater. 121 (1984) 55

[50] P. C. Stangeby and G. M. McCracken, Nucl. Fusion 30 (1990) 1225 


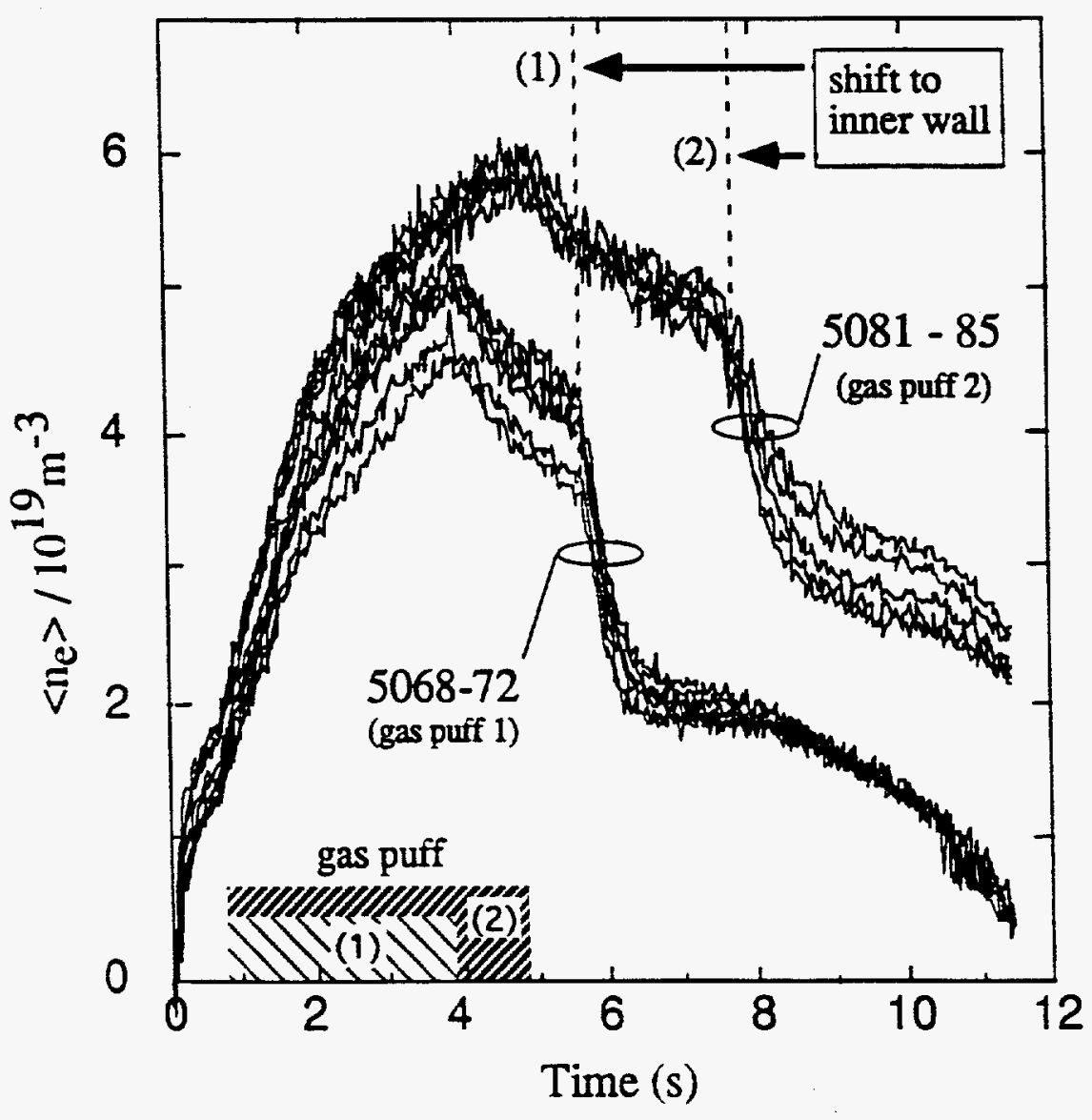

Fig. 1 Density evolution for discharge sequence 5068 - 5985 


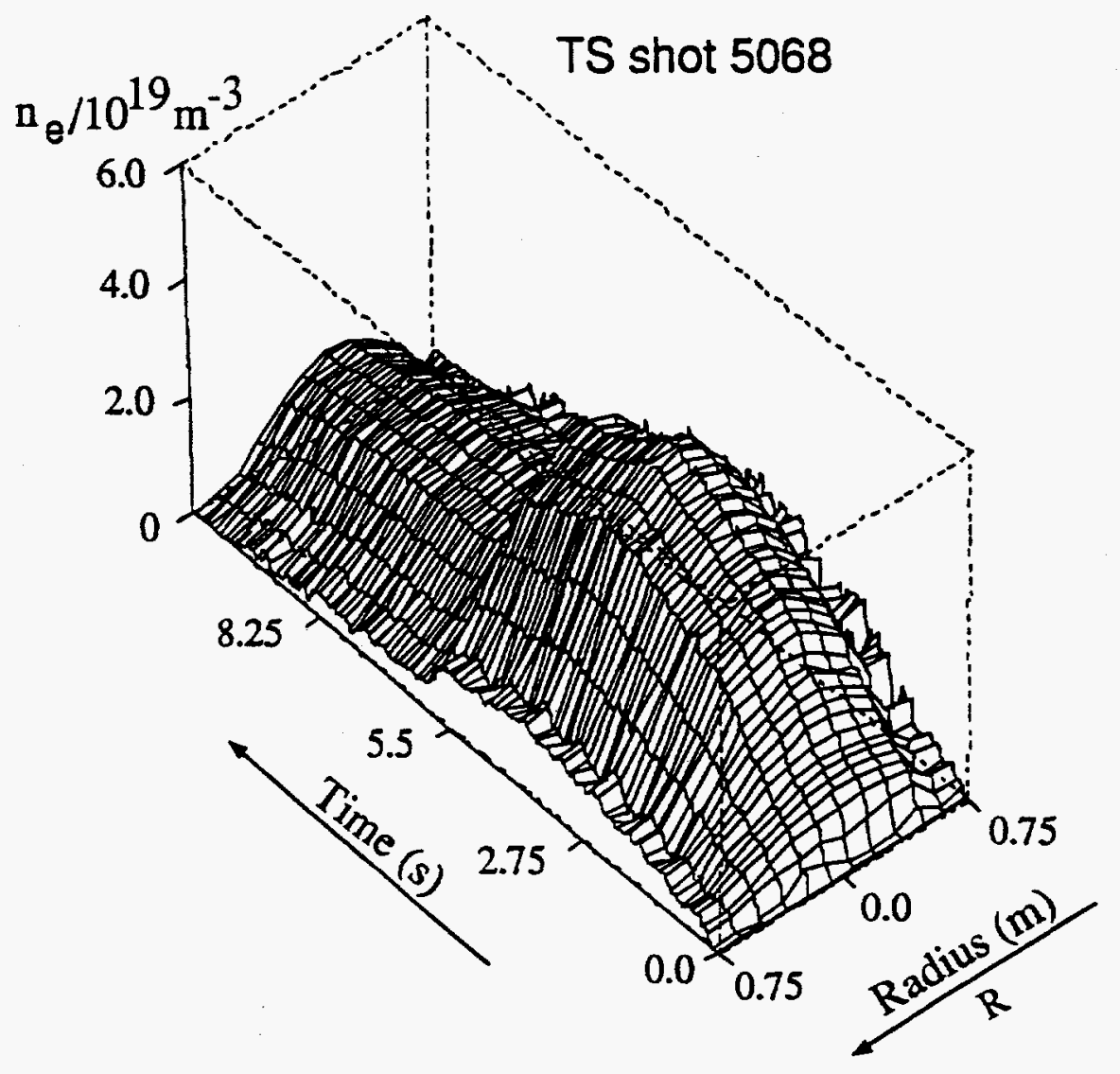

Fig. 2. Electron density spatial profile as a function of time. The plasma is shifted to the inner wall at $t=5.5 \mathrm{~s}$ and the density decay begins at that time. 


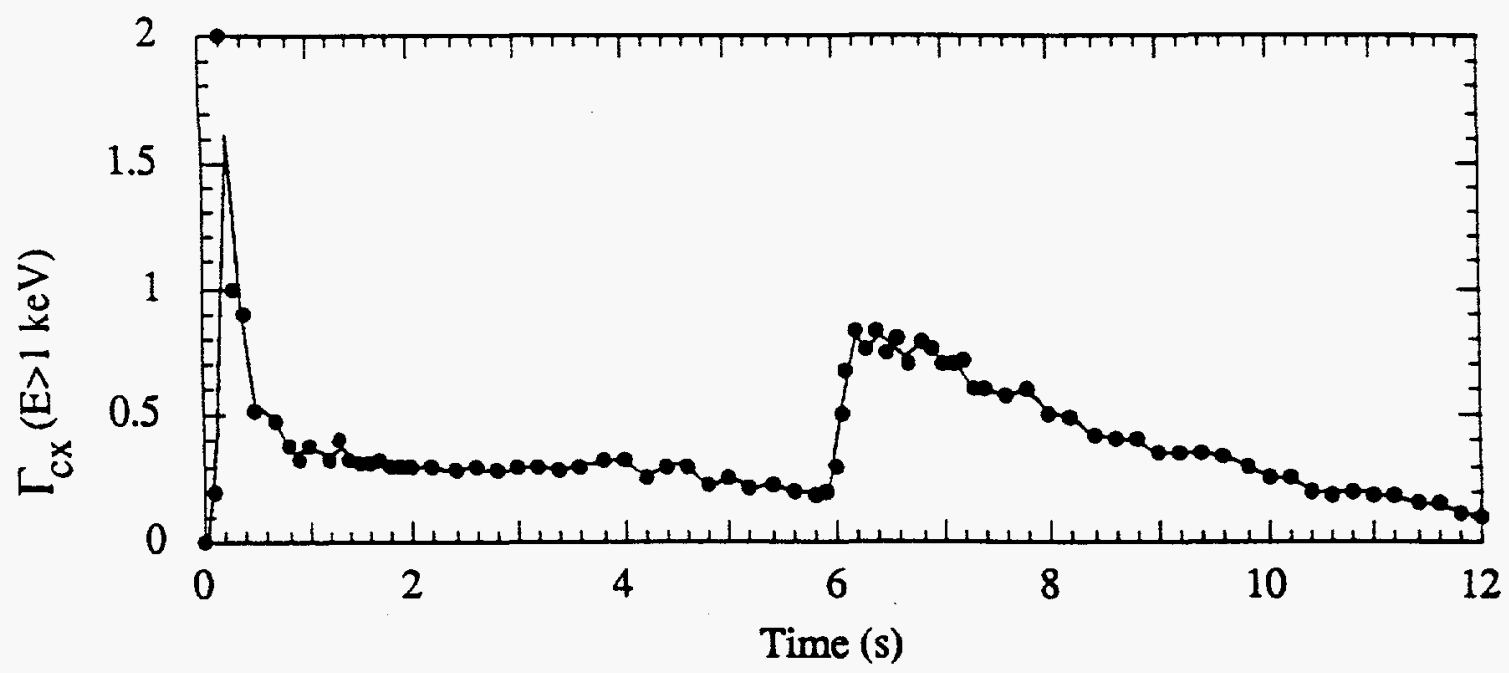

Fig. 3(a). Measured energetic charge exchange flux $(E>1 \mathrm{keV})$; the plasma is shifted to the inboard wall at $t=5.5 \mathrm{~s}$.

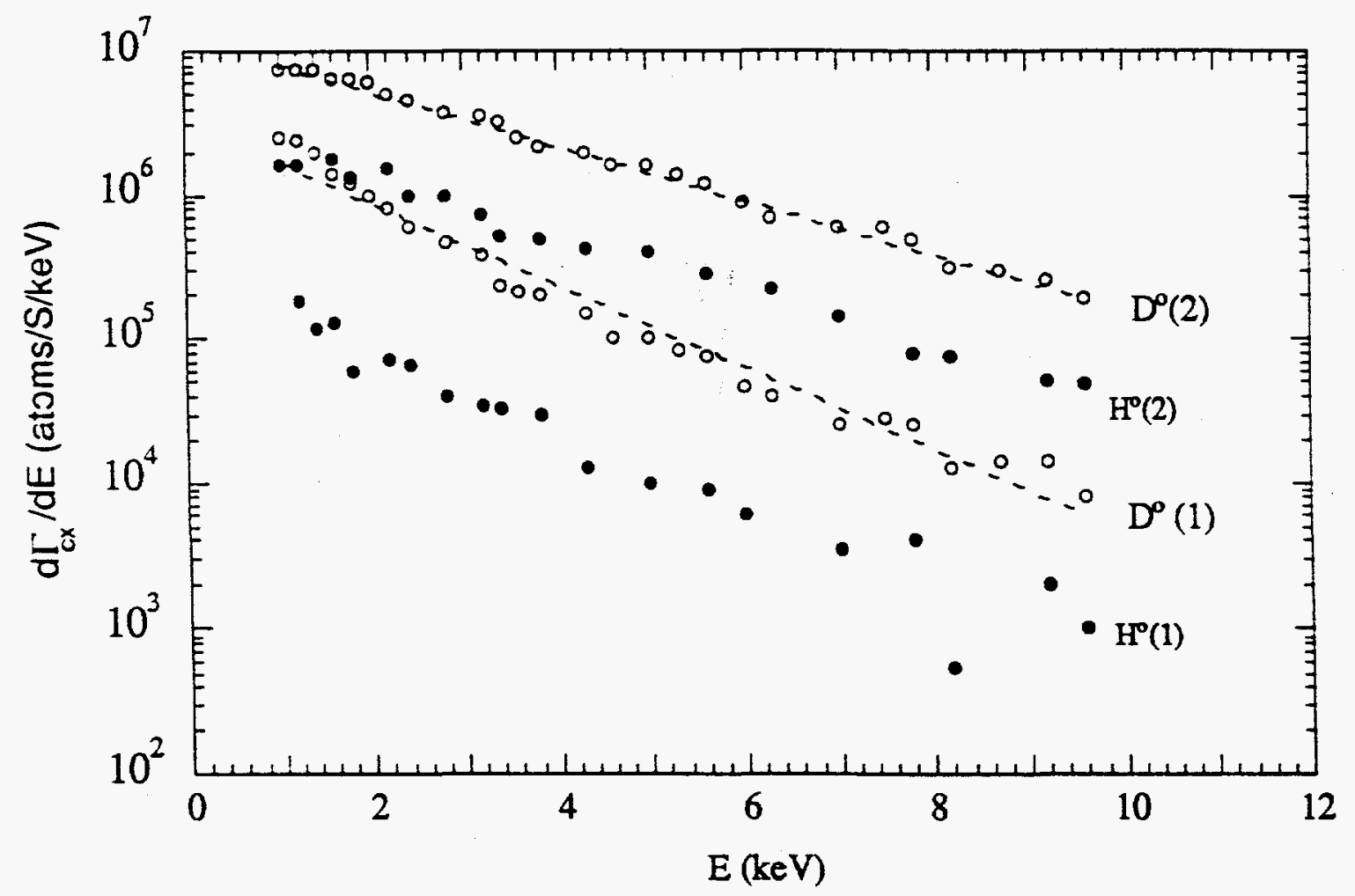

Fig. 3b. Measured charge exchange spectrum at $t=4.3 \mathrm{~s}$ before the plasma is moved to the inner wall (1), and at $t=6.5 \mathrm{~s}$, after the plasma shift to the inner wall (2). 


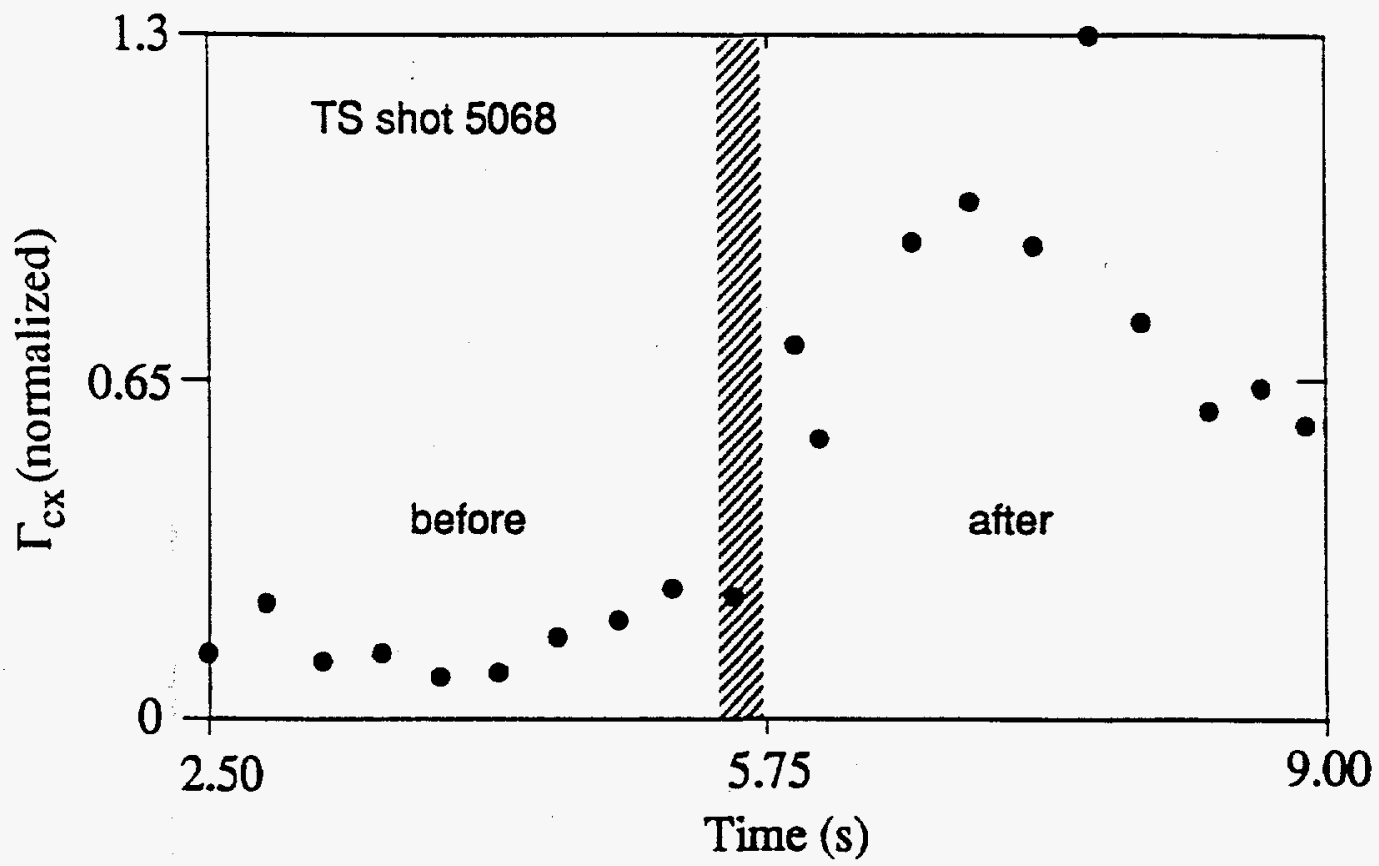

Fig. 4a Calculated energeric charge exchange flux (E>1 keV) as a function of time. The plasma is shifted to the inboard wall at $t=5.5 \mathrm{~s}$.

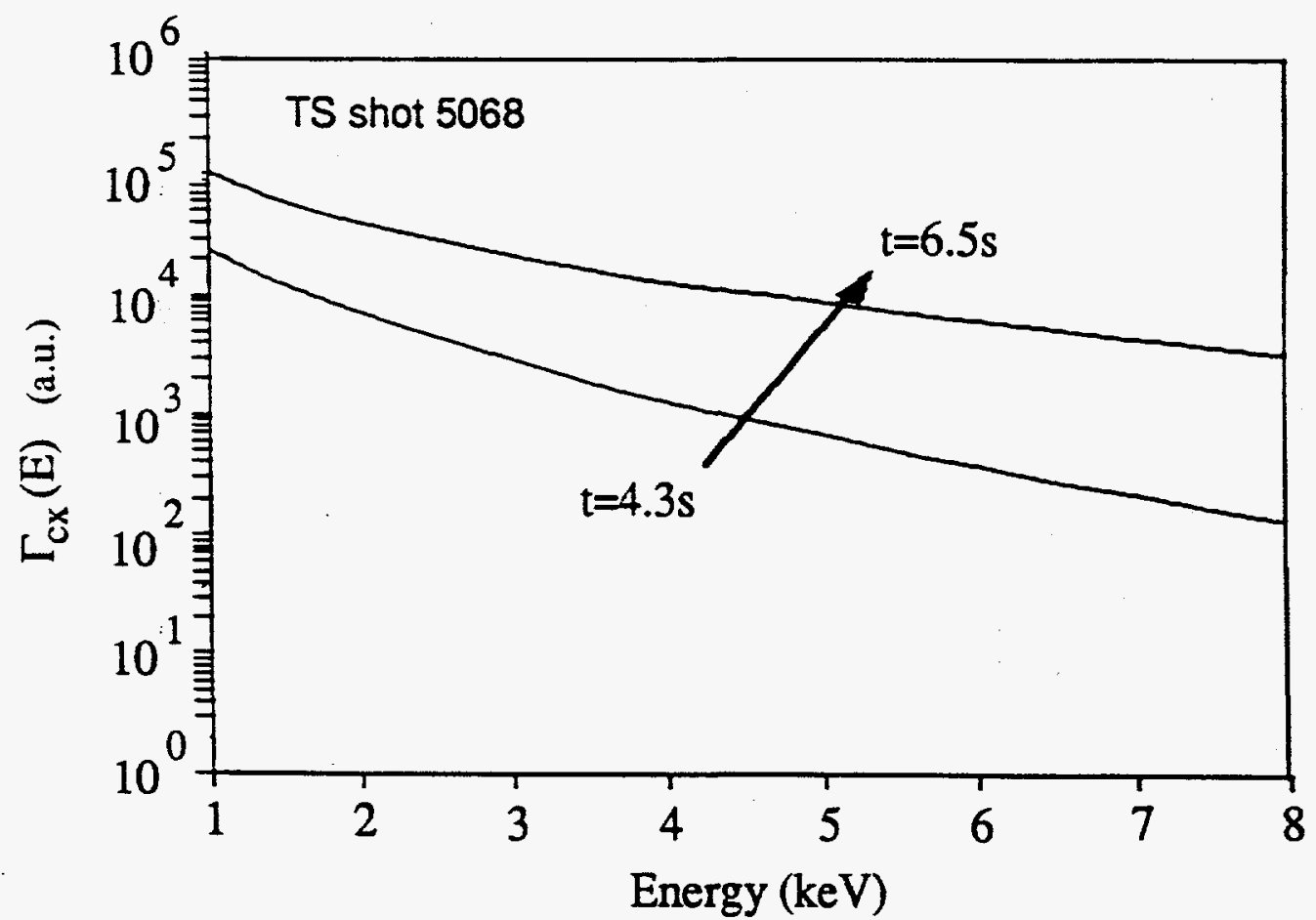

Fig. 4(b) Calculated charge exchange spectrum at $t=4.3 \mathrm{~s}$ before the plasma shift, and at $t=6.5 \mathrm{~s}$, after the plasma is shifted to the inboard wall. 


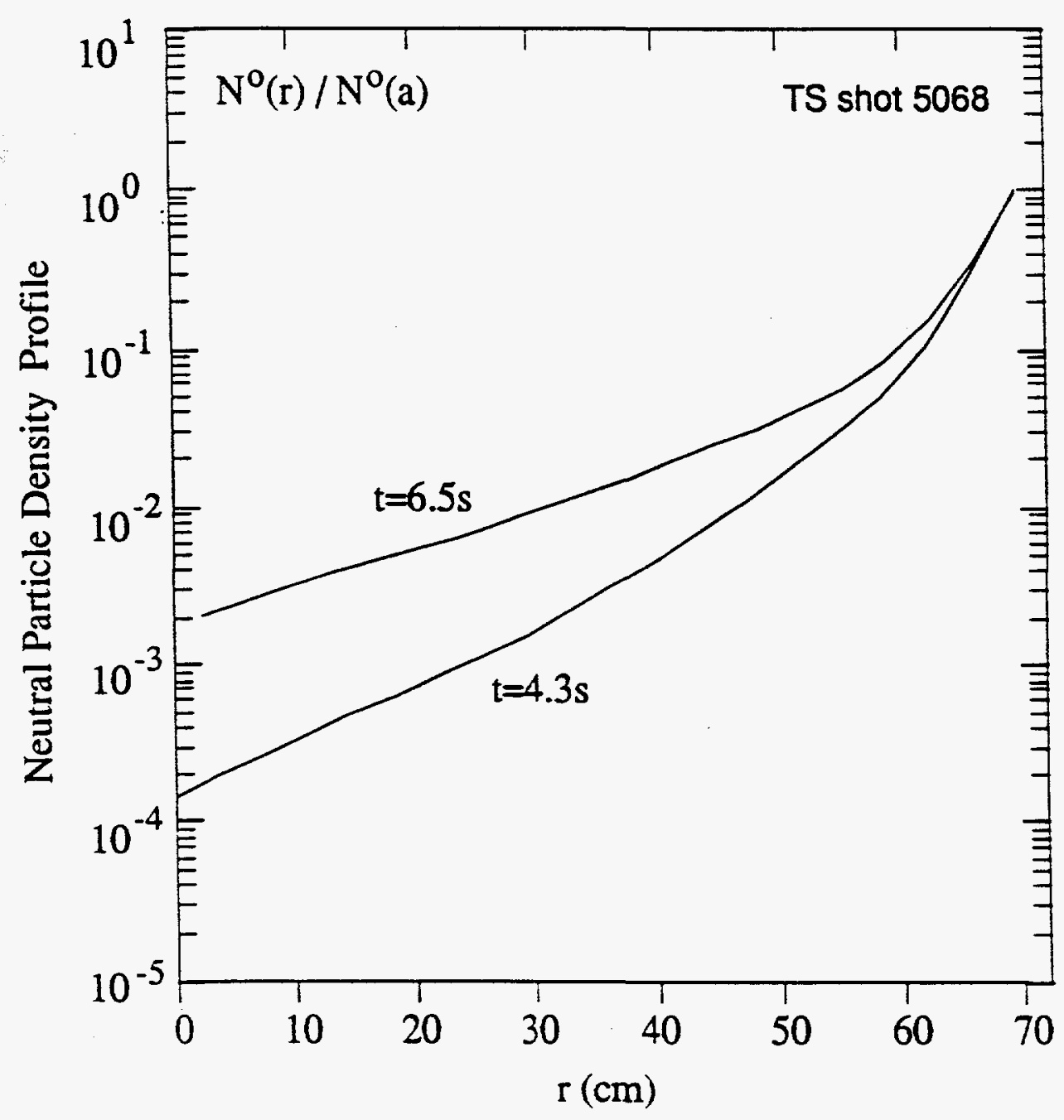

Fig. 5. Calculated spatial distribution of neutral density, normalized to the edge value, before and after the plasma is moved to the inboard wall at $t=5.5 \mathrm{~s}$. 

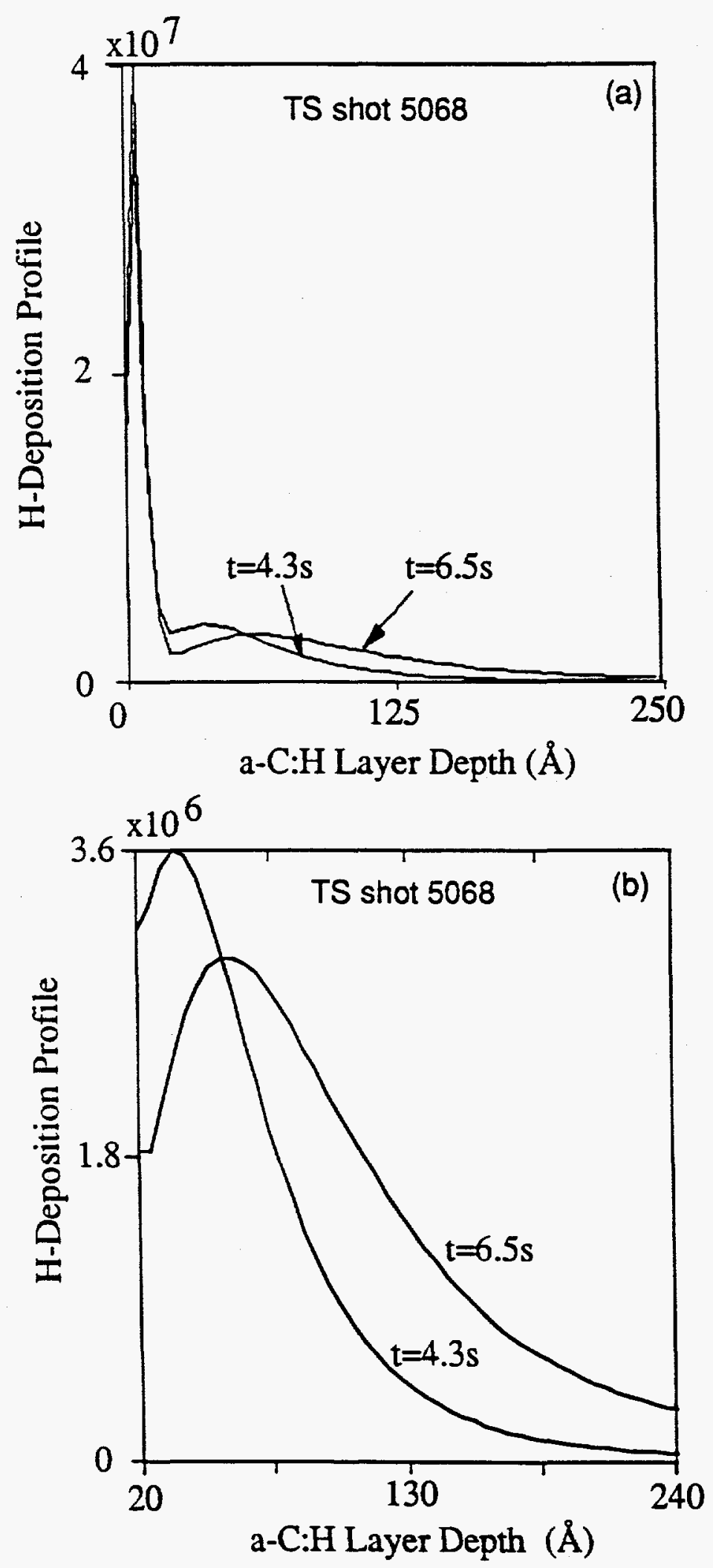

Fig. 6. Wall deposition of charge exchange flux beforeand after the plasma is shifted to the inboard wall: (a) total distribution, (b) expanded view of deep deposition of energetic particles. 


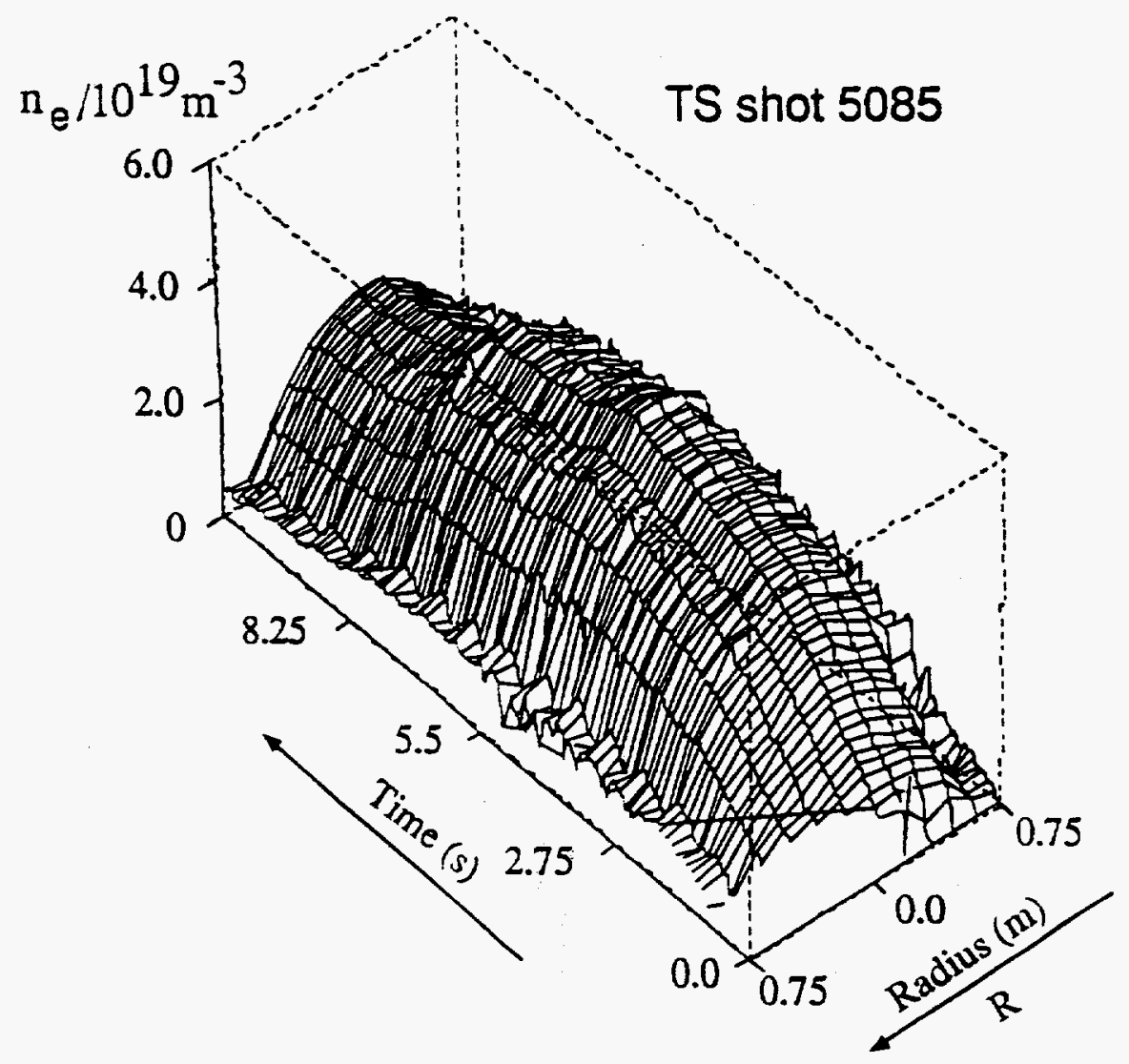

Fig. 7. Electron densiry spatial profile of shot 5085 as a function of time; plasma is shifted to the inboard wall at $t=8 \mathrm{~s}$ 

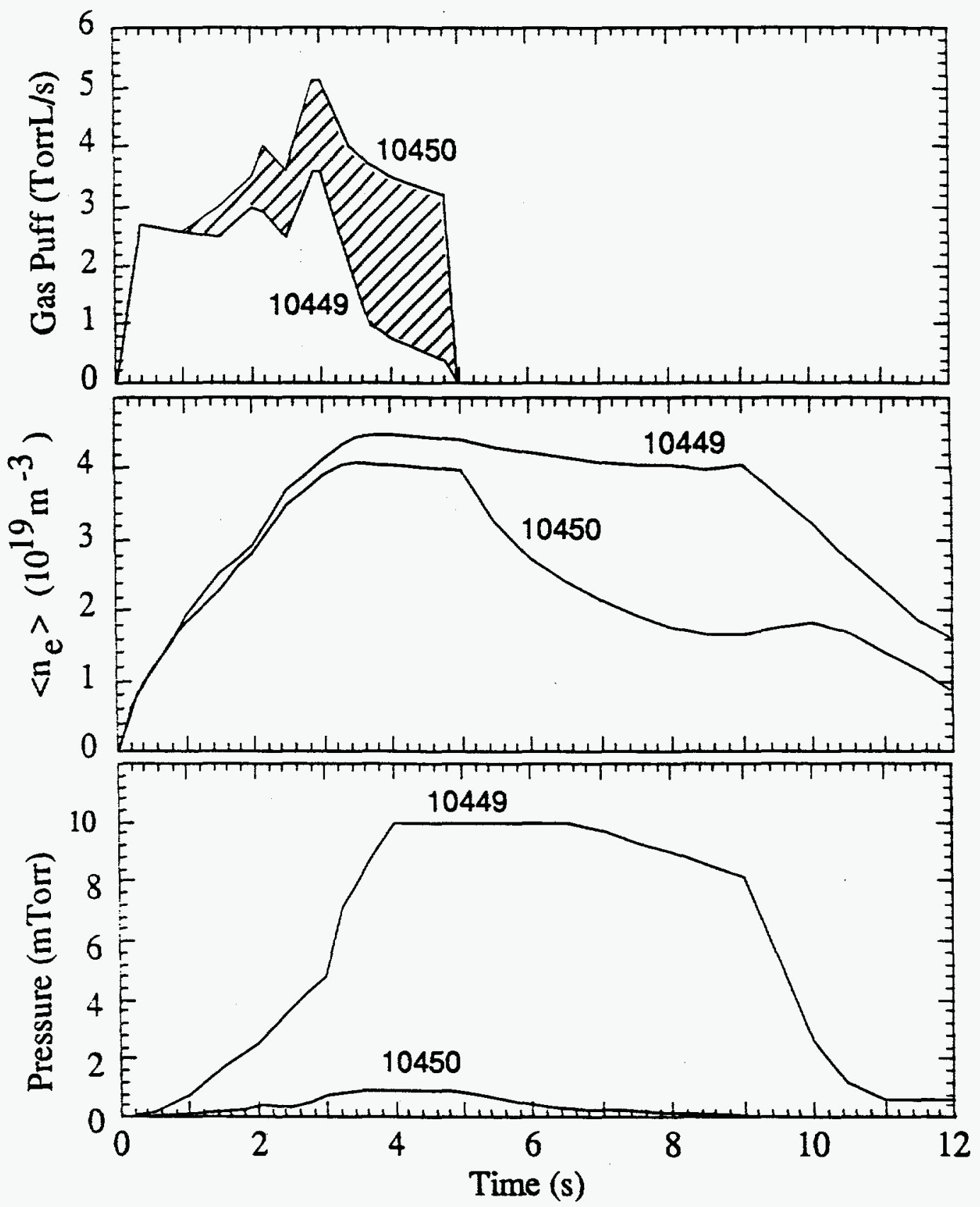

Fig. 8. Comparison of the density evolution in Tore Supra without pumping (10449) andwith pumping (10450) 


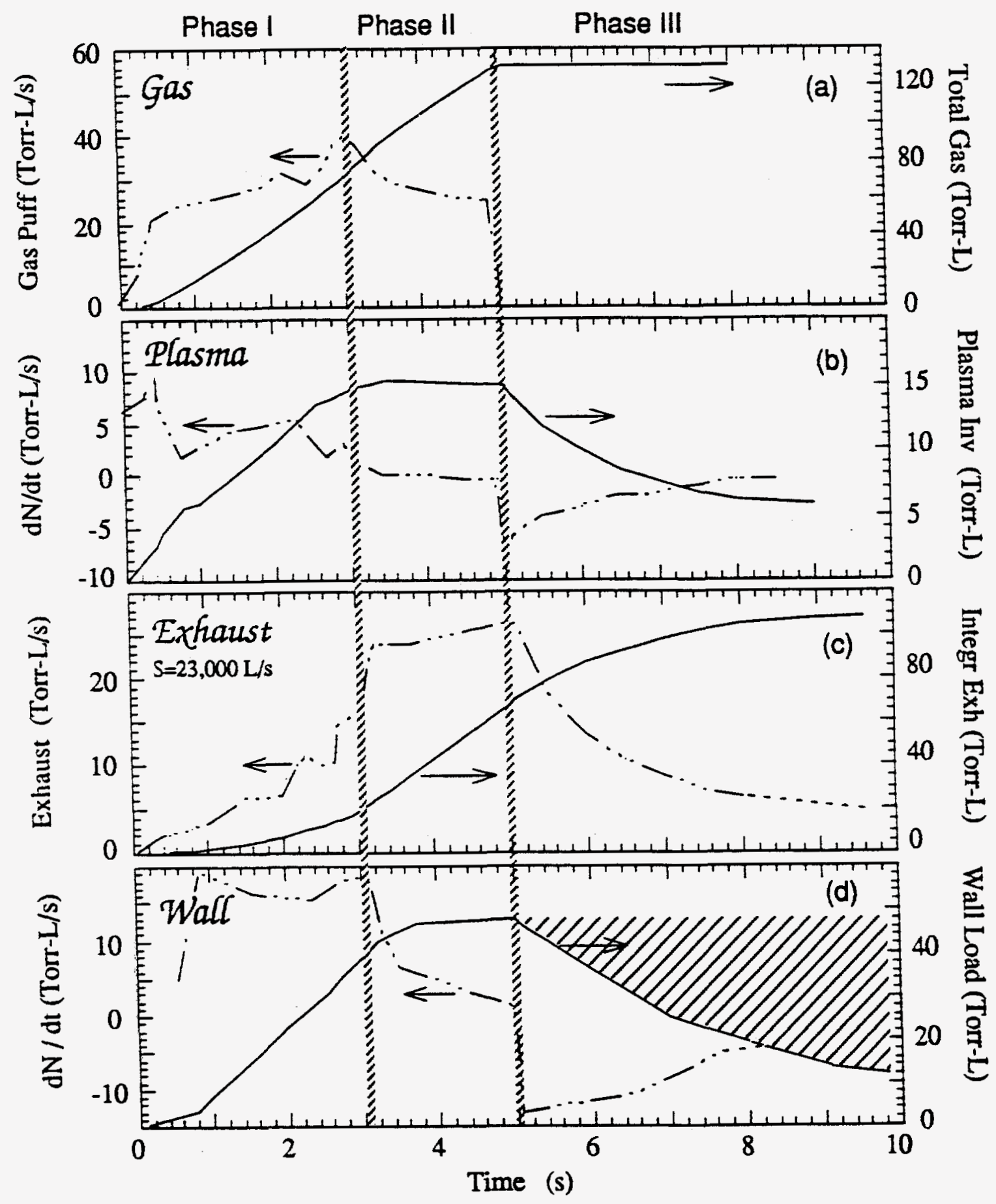

Fig. 9. Particle balance with pump limiter exhaust and gas puffing for shot 10450; (a) gas puff, (b) density evolution, (c) pump limiter exhaust, (d) inferred wall load 


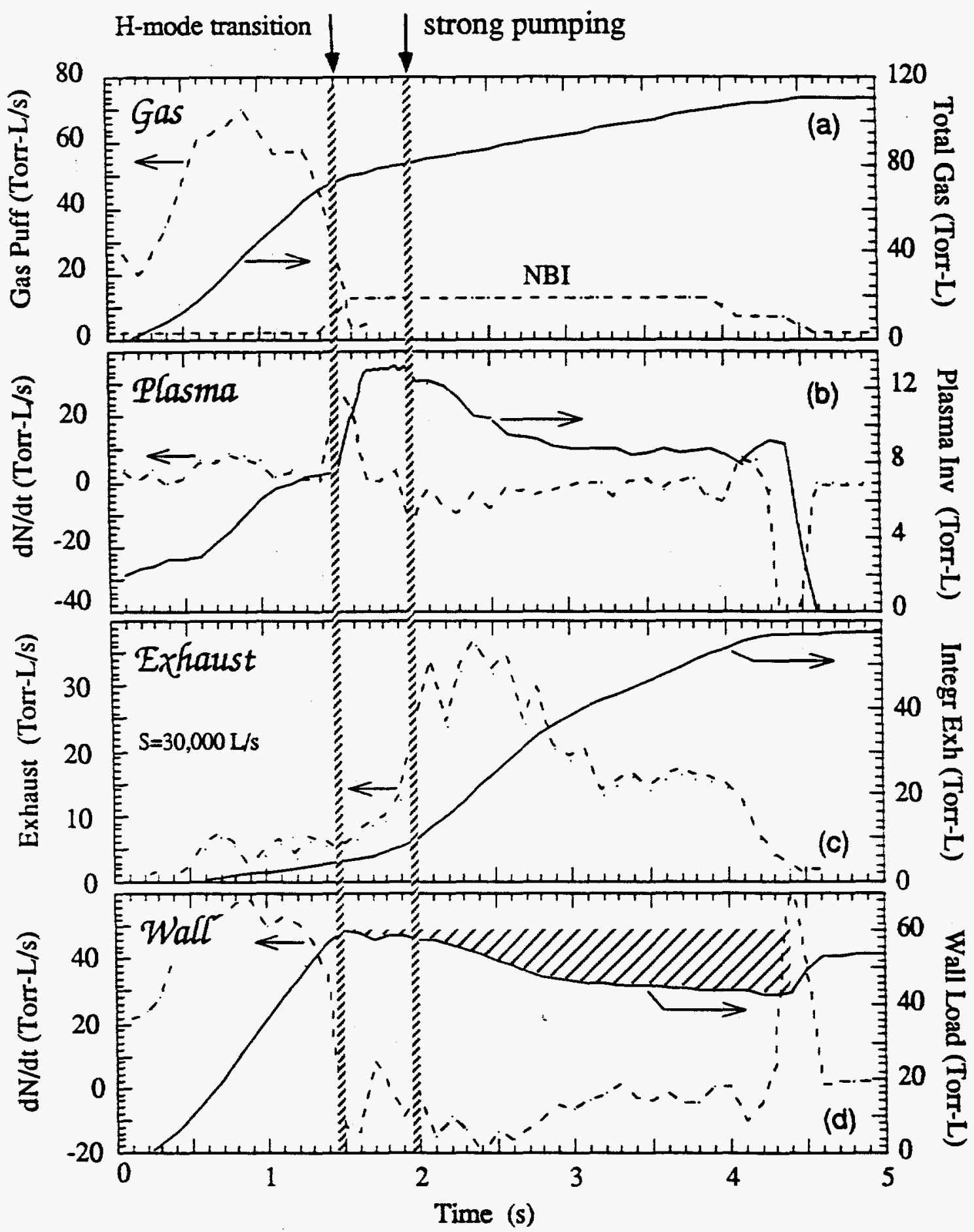

Fig. 10. Particle balance for a divertor discharge in DIII-D (78012): (a) fueling, (b) plasma, (c) exhaust, (d) wall inventory. After the H-mode transition the fueling is supplied by $5 \mathrm{MW}$ of beam injection. During this time, the exhaust rate exceeds the sum of fueling plus plasma decay rate, removing particles from the wall. 


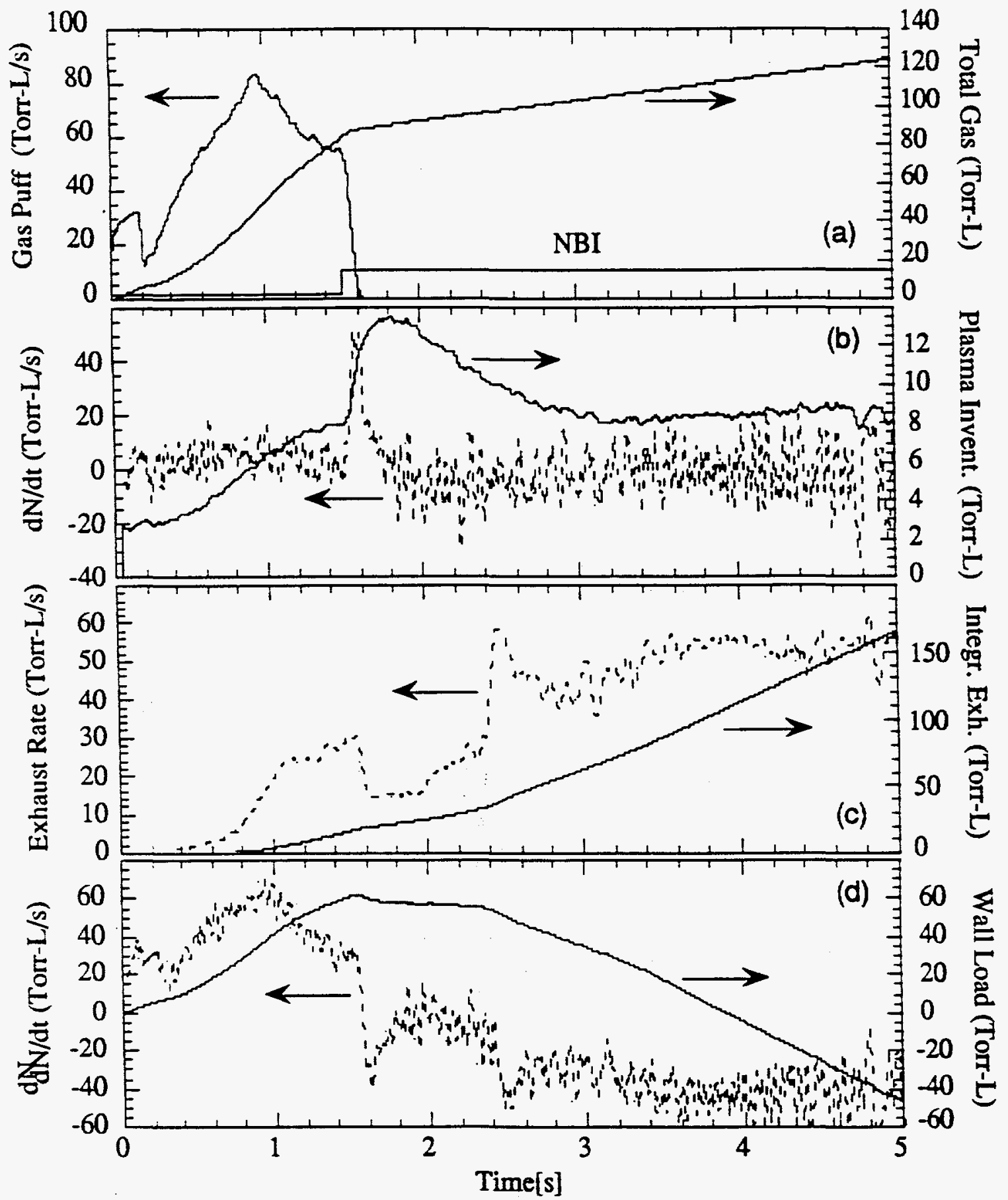

Fig. 11. Particle balance for DIII-D discharge 77155: (a) fueling, (b) plasma inventory, (c) divertor exhaust, (d) wall inventory. The exhaust is strong enough to cause a net depletion of the wall inventory. 

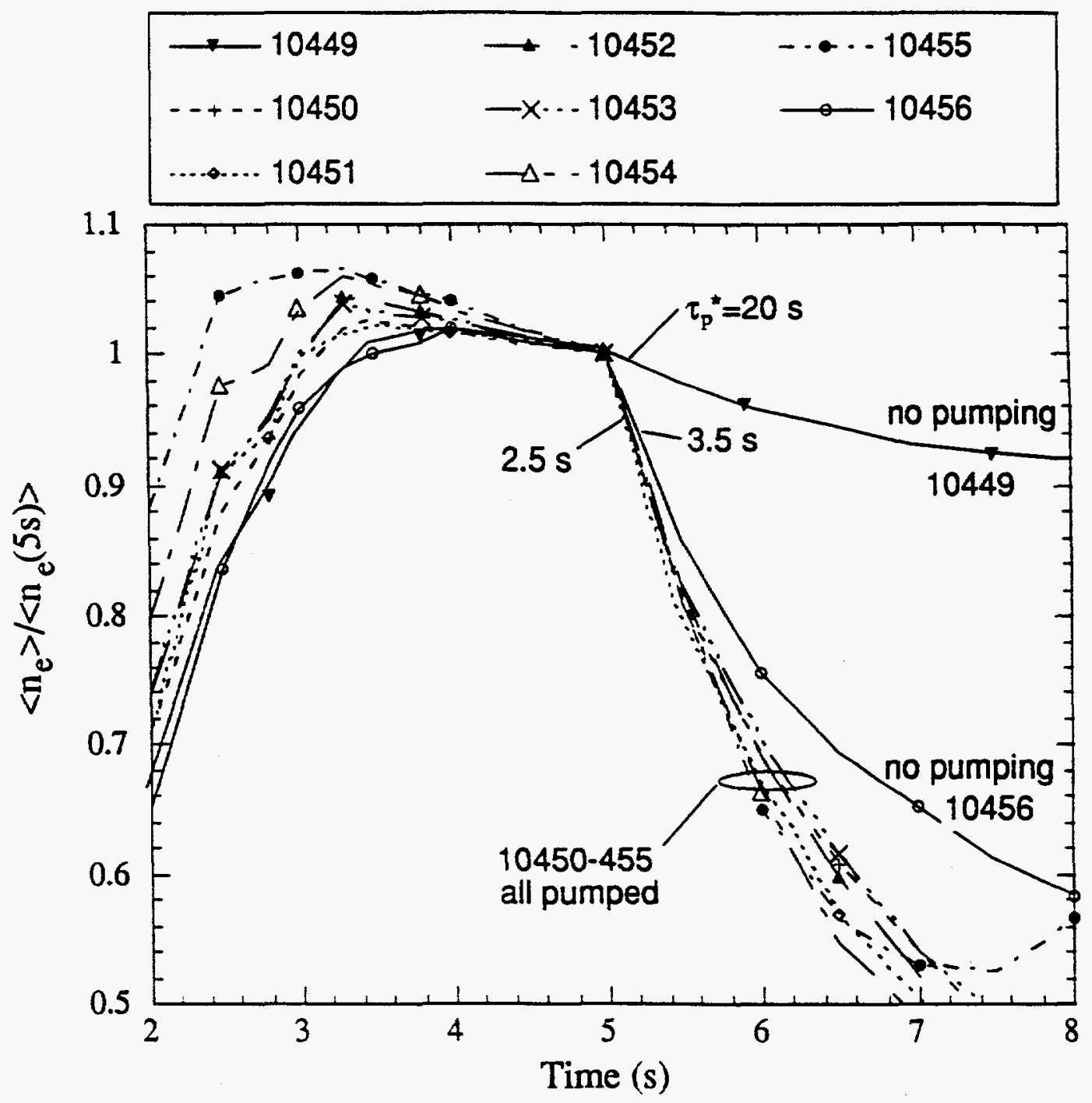

Fig. 12. Series of discharges normalized to the density at $t=5 \mathrm{~s}$, at the termination of the gas puff. 10449 is the last shot before pumping, 10450-10455 were all pumped, and 10456 is again without pumping. 


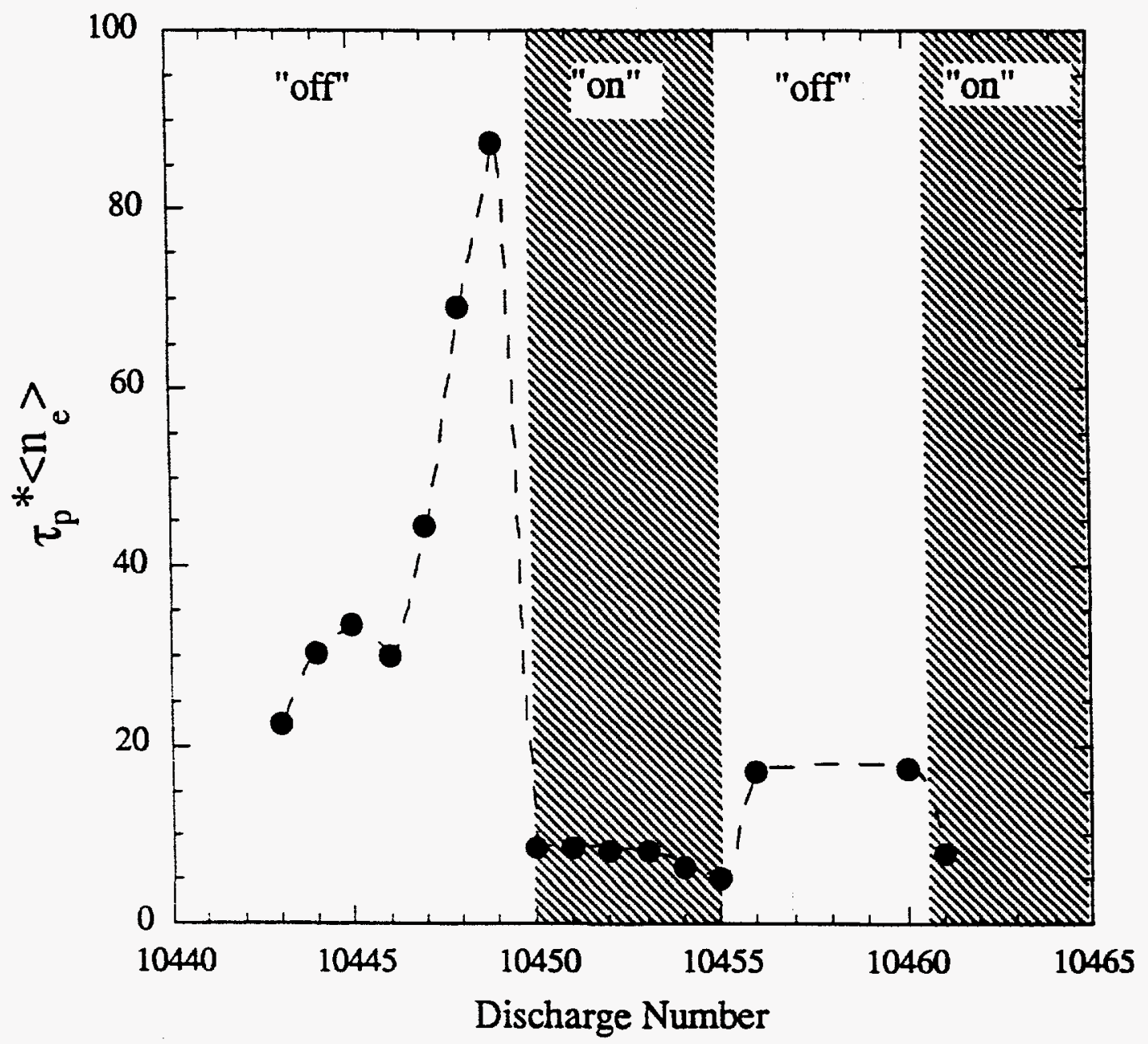

Fig. 13. Evolution of normalized $\tau_{p} *$ as a function of shot number. The pump limiter was pumping for shots 10450-10455 and 10461. 


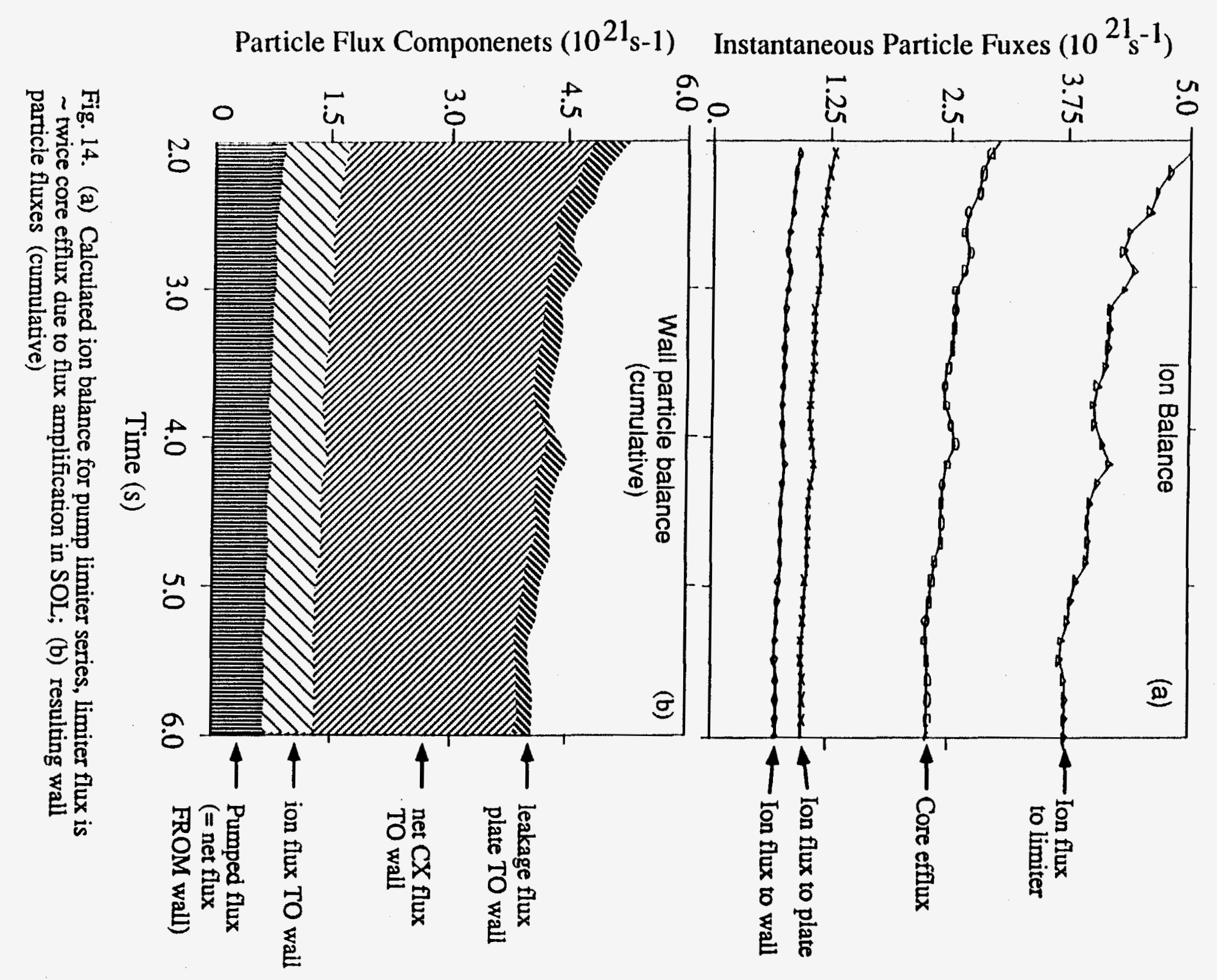




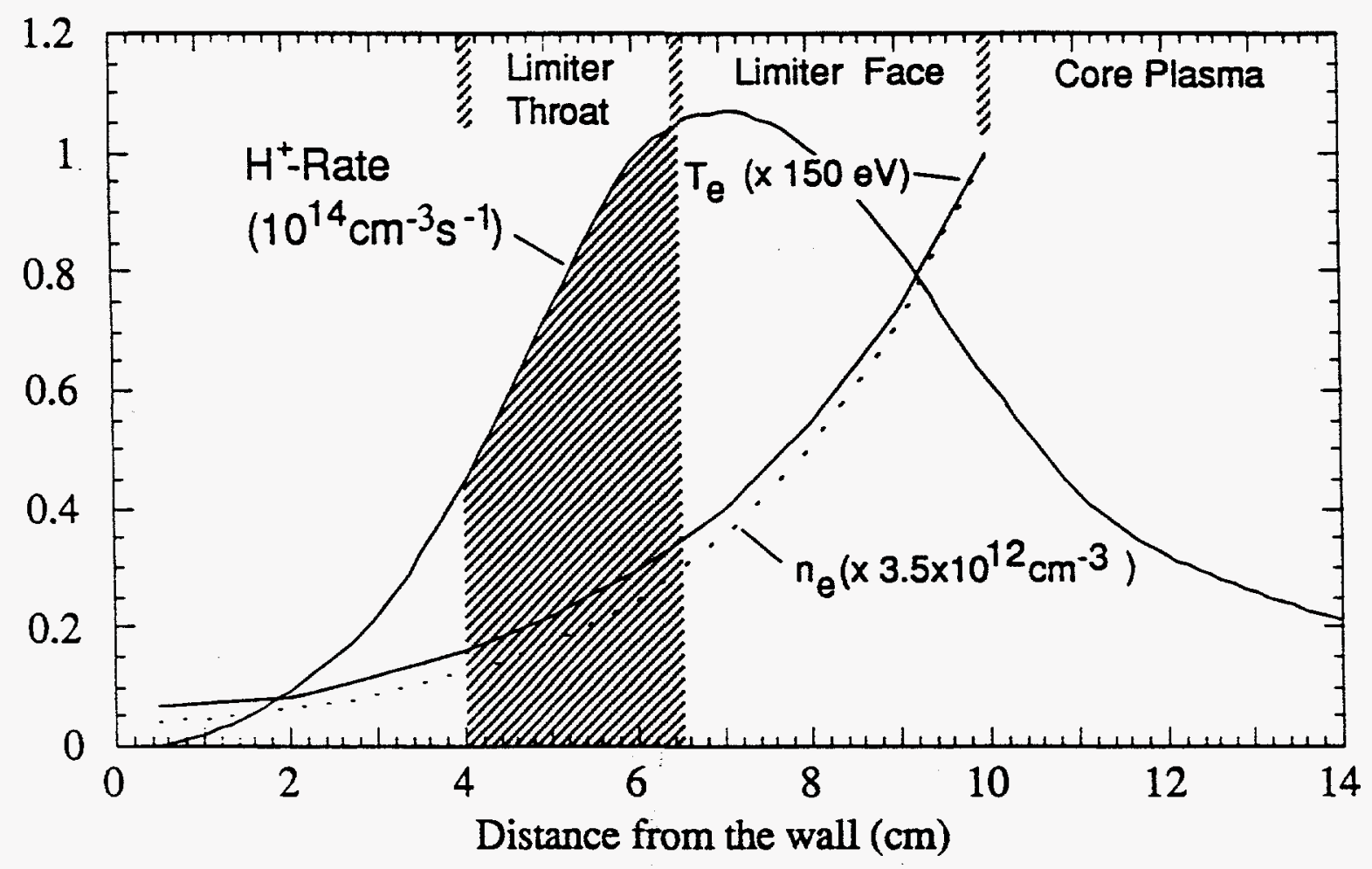

Fig. 15. Effect of the ionizing SOL: electron temperature and density calculated with Stangeby's model and DEGAS; $\mathrm{H}^{+}$-rate distribution calculated with DEGAS. The shaded fraction has direct access to the pump limiter throat.

\section{DISCLAIMER}

This report was prepared as an account of work sponsored by an agency of the United States Government. Neither the United States Government nor any agency thereof, nor any of their employees, makes any warranty, express or implied, or assumes any legal liability or responsibility for the accuracy, completeness, or usefulness of any information, apparatus, product, or process disclosed, or represents that its use would not infringe privately owned rights. Reference herein to any specific commercial product, process, or service by trade name, trademark, manufacturer, or otherwise does not necessarily constitute or imply its endorsement, recommendation, or favoring by the United States Government or any agency thereof. The views and opinions of authors expressed herein do not necessarily state or reflect those of the United States Government or any agency thereof. 